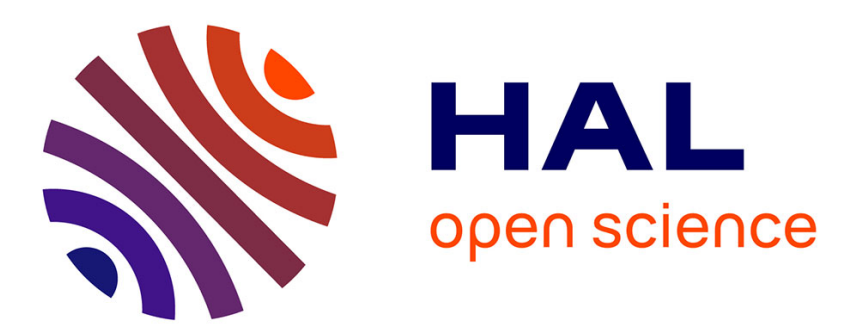

\title{
Understanding the Time-Dependent Effective Diffusion Coefficient Measured by Diffusion MRI: the Intra-Cellular Case
}

\author{
Houssem Haddar, Jing-Rebecca Li, Simona Schiavi
}

\section{To cite this version:}

Houssem Haddar, Jing-Rebecca Li, Simona Schiavi. Understanding the Time-Dependent Effective Diffusion Coefficient Measured by Diffusion MRI: the Intra-Cellular Case. SIAM Journal on Applied Mathematics, 2017. hal-01421928

\section{HAL Id: hal-01421928 \\ https://hal.inria.fr/hal-01421928}

Submitted on 23 Dec 2016

HAL is a multi-disciplinary open access archive for the deposit and dissemination of scientific research documents, whether they are published or not. The documents may come from teaching and research institutions in France or abroad, or from public or private research centers.
L'archive ouverte pluridisciplinaire HAL, est destinée au dépôt et à la diffusion de documents scientifiques de niveau recherche, publiés ou non, émanant des établissements d'enseignement et de recherche français ou étrangers, des laboratoires publics ou privés. 


\title{
UNDERSTANDING THE TIME-DEPENDENT EFFECTIVE DIFFUSION COEFFICIENT MEASURED BY DIFFUSION MRI: THE INTRA-CELLULAR CASE*
}

\author{
HOUSSEM HADDAR ${ }^{\dagger}$, JING-REBECCA LI ${ }^{\dagger}$, AND SIMONA SCHIAVI ${ }^{\dagger}$
}

Abstract. Diffusion Magnetic Resonance Imaging (dMRI) can be used to measure a timedependent effective diffusion coefficient that can in turn reveal information about the tissue geometry. Recently a mathematical model for the time-dependent effective diffusion coefficient was obtained using homogenization techniques after imposing a certain scaling relationship for the time, the biological cell membrane permeability, the diffusion-encoding magnetic field gradient strength, and a periodicity length of the cellular geometry. With this choice of the scaling of the physical parameters, the effective diffusion coefficient of the medium can be computed after solving a diffusion equation subject to a time-dependent Neumann boundary condition, independently in the biological cells and in the extra-cellular space. In this paper, we analyze this new model, which we call the $\mathrm{H}-A D C$ model, in the case of finite domains, which is relevant to diffusion inside biological cells. We use both the eigenfunction expansion and the single layer potential representation for the solution of the above mentioned diffusion equation to obtain analytical expressions for the effective diffusion coefficient in different diffusion time regimes. These expressions are validated using numerical simulations in two dimensions.

Key words. Diffusion MRI; time-dependent $A D C$; homogenization; effective medium;

AMS subject classifications. 35B27, 35Q99, 65M32, 65Z05

1. Introduction. Diffusion Magnetic Resonance Imaging (dMRI) encodes water displacement due to diffusion via the application of diffusion-encoding gradient pulses and is a powerful tool to obtain information on the tissue microstructure. A major application has been in detecting acute cerebral ischemia minutes after stroke [28, 42]. DMRI has been used to detect and differentiate a wide range of physiological and pathological conditions in the brain, including tumors $[25,37,40]$ and myelination abnormalities (for a review, see [21]). It also has been used to study brain connectivity (for a review, see [20]) and in functional imaging [22] as well as in cardiac applications $[5,6,35]$.

In particular, we are interested in an important quantity measured by dMRI called the "Apparent Diffusion Coefficient" $(A D C)$ which is based on a measure of the mean diffusion displacement inside an imaging voxel. The mean squared displacment of spins during a diffusion time $t_{D}$ is defined as:

$$
M S D\left(t_{D}\right) \equiv \frac{1}{\int_{\mathbf{x}_{0}} \rho\left(\mathbf{x}_{0}\right) d \mathbf{x}_{0}} \int_{\mathbf{x}_{0}} \int_{\mathbf{x}} \rho\left(\mathbf{x}_{0}\right)\left(\left(\mathbf{x}-\mathbf{x}_{0}\right) \cdot \mathbf{u}_{\mathbf{g}}\right)^{2} u\left(\mathbf{x}, \mathbf{x}_{0}, t_{D}\right) d \mathbf{x} d \mathbf{x}_{0} .
$$

where $u\left(\mathbf{x}, \mathbf{x}_{0}, t_{D}\right)$ is the proportion of spins starting at $\mathbf{x}_{0}$ when $t=0$ ending up at position $\mathbf{x}$ after a time $t_{D}, \rho\left(\mathbf{x}_{0}\right)$ is the density of spins at $\mathbf{x}_{0}$, and $\mathbf{u}_{\mathbf{g}}$ is a unit vector in $\mathbb{R}^{3}$ ( $\mathbf{u}_{\mathrm{g}}$ is called the diffusion-encoding direction). In the same vein, we can define an effective diffusion coefficient in the direction $\mathbf{u}_{\mathbf{g}}$ by the following expression:

$$
\frac{1}{2 t_{D}} M S D\left(t_{D}\right)
$$

\footnotetext{
*Submitted to the editors December, 10th 2016.

Funding: ...

†INRIA- CMAP, École Polytechnique, Paris-Saclay (houssem.haddar@inria.fr, jingrebecca.li@inria.fr, simona.schiavi@polytechnique.edu).
} 
Since the mean squared displacement in a heterogeneous medium is not necessarily linear in $t_{D}$, effective diffusion coefficient typically depends on $t_{D}$.

The $M S D$ can be measured by dMRI using a sequence of magnetic field gradient pulses (called a diffusion-encoding sequence). A commonly used sequence is the Pulse Gradient Spin Echo (PGSE) experiment [36]. In this paper, we will focus on the PGSE time profile to simplify the presentation.

The PGSE sequence contains two rectangular pulses of the diffusion-encoding gradient magnetic field $B_{\text {diff }}=g \mathbf{u}_{\mathbf{g}} \cdot \mathbf{x}$, where $g$ is the strength of the gradient and $\mathbf{u}_{\mathbf{g}}$ is the gradient direction. Each pulse has a duration $\delta$, with the delay between the start of the two pulses denoted $\Delta$, and there is also a radio-frequency ( $\mathrm{RF}$ ) pulse to affect a 180 degree spin reversal between the pulses (see Figure 1).

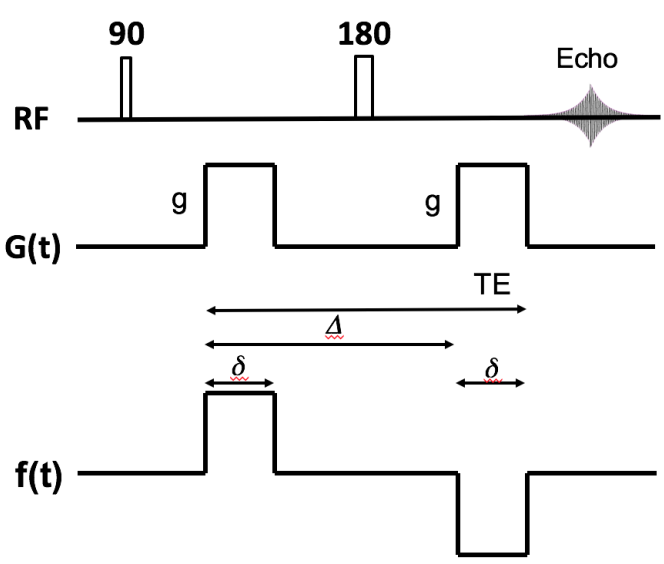

Fig. 1: The Pulsed Gradient Spin Echo (PGSE) sequence. The effective sequence time profile shown here takes into account the 180 degree pulse.

In the ideal case, where the pulse duration is very short compared to the delay between the pulses, $\delta \ll \Delta$, called the narrow pulse case, it is easy to relate the magnetization of spins to the diffusion propagator $u\left(\mathbf{x}, \mathbf{x}_{0}, t_{D}\right)$. Let us consider spins initially located at $\mathbf{x}_{0}$. After the first pulse, the complex phase of these spins is $e^{i \delta \gamma \mathbf{g} \cdot \mathbf{x}_{0}}$, where $\gamma=42.576 \mathrm{MHz} /$ Tesla is the gyro-magnetic ratio of the water proton. Because the gradient magnetic field is turned off after the first pulse, the spins move but the phase of the spins does not change. The phase remains $e^{i \delta \gamma \mathbf{g} \cdot \mathbf{x}_{0}}$, until the application of the RF pulse resulting in a 180 degree spin reversal. After the 180 degree RF pulse, the complex phase becomes $e^{-i \delta \gamma \mathbf{g} \cdot \mathbf{x}_{0}}$. Again, spins move but the phase of the spins stays the same until the application of the second pulse, after which the complex phase due to spins ending up at position $\mathbf{x}_{f}$ becomes $e^{i \delta \gamma \mathbf{g} \cdot\left(\mathbf{x}_{f}-\mathbf{x}_{0}\right)}$. So the magnetization at position $\mathbf{x}$ and the echo time $T E$, which is the time that the MRI signal is acquired, some time after the end of the second pulse (i.e. $T E \geq \Delta+\delta$ ), is:

$$
M(\mathbf{x}, T E) \approx \int_{\mathbf{x}_{0}} \rho\left(\mathbf{x}_{0}\right) u\left(\mathbf{x}, \mathbf{x}_{0}, \Delta\right) e^{i \delta \gamma g \mathbf{u}_{\mathbf{g}} \cdot\left(\mathbf{x}-\mathbf{x}_{0}\right)} d \mathbf{x}_{0}
$$

where we used the assumption that $\delta \ll \Delta$. The dMRI signal $S$ is the total water 
proton magnetization in an imaging voxel $V$ :

$$
S=\int_{\mathbf{x} \in V} M(\mathbf{x}, T E) d \mathbf{x} .
$$

Because the diffusion displacement is usually much shorter than the size of the imaging voxel size we can ignore spins that enter and leave the voxel during the signal acquisition and thus take domain of integration in Eq. (4) to be $\mathbb{R}^{3}$. Using properties of the Fourier transform, we obtain

$$
\left.\frac{\partial \frac{S}{S_{0}}}{\partial(\delta \gamma g)^{2}}\right|_{\delta \gamma g=0} \approx M S D(\Delta),
$$

in the case of the narrow pulse PGSE sequence, where $S_{0}$ is the signal at $g=0$ (a derivation of this statement can be found in [23]).

Without the narrow pulse assumption, Eq. (3) does not hold exactly. Rather, $M(\mathbf{x}, t)$ is governed by the Bloch-Torrey equation, which is a complex-valued diffusive PDE:

(6)

$$
\begin{cases}\frac{\partial}{\partial t} M(\mathbf{x}, t)=-\iota \gamma g \mathbf{u}_{\mathbf{g}} \cdot \mathbf{x} f(t) M(\mathbf{x}, t)+\operatorname{div}\left(\mathcal{D}_{0}(\mathbf{x}) \nabla M(\mathbf{x}, t)\right) & \text { in } \bigcup \Omega_{j} \times[0, T E] \\ \llbracket \mathcal{D}_{0} \nabla M \cdot \nu \rrbracket_{\Gamma_{i j}}=0 & \text { on } \Gamma_{i j} \times[0, T E] \\ \left.\mathcal{D}_{0} \nabla M \cdot \nu\right|_{\Gamma_{i j}}=\kappa \llbracket M \rrbracket_{\Gamma_{i j}} & \text { on } \Gamma_{i j} \times[0, T E] \\ M(\cdot, 0)=\rho & \text { in } \bigcup \Omega_{j}\end{cases}
$$

where $\Omega_{0}$ is the extra-cellular space and each of $\Omega_{j}, j=1, \cdots, N$, is a biological cell. The vector $\nu$ is the exterior normal to the biological cells; $\llbracket \cdot \rrbracket_{\Gamma_{i j}}, i, j=0, \cdots, N, i \neq j$, is the jump (the limit value in compartment $i$ minus the limit value in compartment $j$ ) on $\Gamma_{i j}$, the interface between $\Omega_{i}$ and $\Omega_{j} ; \kappa$ is the membrane permeability coefficient; $\iota$ is the imaginary unit. The function $f(t)$ gives the normalized time profile of the diffusion-encoding magnetic field gradient pulses. For the classic Pulsed Gradient Spin Echo (PGSE) sequence [36], simplified to include only the parameters relevant to diffusion (the imaging gradients are ignored),

$$
f(t)= \begin{cases}1 & t_{s}<t \leq t_{s}+\delta \\ 0 & t_{s}+\delta<t \leq t_{s}+\Delta \\ -1 & t_{s}+\Delta<t \leq t_{s}+\Delta+\delta \\ 0 & \text { elsewhere }\end{cases}
$$

where $t_{s}$ is the start of the first pulse and we made $f(t)$ negative in the second pulse to include the effect of the 180 degree spin reversal between the pulses. For simplicity, since $t_{s}$ does not play a role in the results of this paper, we set $t_{s} \rightarrow 0$. For the same reason, we set $T E=\delta+\Delta$ in this paper.

We note that the Bloch-Torrey equation needs to be supplemented by additional boundary conditions on the sides of the imaging voxel. For example, periodic boundary conditions on the boundary of the voxel would be an acceptable choice.

In the case of unrestricted diffusion in a homogeneous medium with a diffusion coefficient $\mathcal{D}_{0}$, the integral of the solution of the Bloch-Torrey equation, in other words, the total magnetization, takes the following exponential form $[4,16]$ :

$$
S=S_{0} e^{-\mathcal{D}_{0} b},
$$


with the $b$-value defined as:

$$
b \equiv \gamma^{2} g^{2} \int_{0}^{T E} F(t)^{2} d t,
$$

where

$$
F(t) \equiv \int_{0}^{t} f(s) d s .
$$

In particular, for the PGSE sequence,

$$
F(t)= \begin{cases}t & t_{s}<t \leq t_{s}+\delta \\ \delta & t_{s}+\delta<t \leq t_{s}+\Delta, \\ \Delta+\delta-t & t_{s}+\Delta<t \leq t_{s}+\Delta+\delta, \\ 0 & \text { elsewhere }\end{cases}
$$

To adapt the definition of the effective diffusion coefficient to the non-narrow pulse case, we make the following mathematical definition:

$$
D_{\mathbf{u}_{\mathbf{g}}}^{\mathrm{eff}} \equiv-\left.\frac{1}{\gamma^{2} \int_{0}^{T E} F(t)^{2} d t} \frac{\partial}{\partial g^{2}} \ln \left(\frac{S}{S_{0}}\right)\right|_{g=0} .
$$

In the dMRI community, the above quantity is fitted using the experimental MRI signal at several $b$ values and the obtained value is called the "apparent diffusion coefficient" $(A D C)$. The $A D C$ is widely used medical applications, for instance, $A D C$ maps of brain have been used to identify tumours (see [21, 41]). The $D_{\mathbf{u}_{\mathbf{g}}}^{\text {eff }}$ defined in the above formula depends on the gradient direction $\mathbf{u}_{\mathbf{g}}$ and the temporal profile $f(t)$ but not on the gradient amplitude. In this paper, with the phrase "diffusion time-dependent" we actually mean dependent on $\Delta$ and $\delta$.

The motivation of our work is the experimentally observed phenomenon (see [33] and the references contained there) that the $A D C$ depends on $\Delta$ (and $\delta$ in the nonnarrow pulse case), leading to the need to characterize the time-dependent $A D C$ in terms of tissue-related quantities over a wide range of diffusion time regimes. The ultimate goal is of course the estimation of these tissue-related quantities from the measured dMRI signal.

In this paper, we focus on the case of finite domains, where the membrane permeability is small enough to have negligible effect on the effective diffusion coefficient, which is related to the first order moment of the dMRI signal in the $b$-value (Eq. 12). We note that this does not exclude the permeability from having an effect on the higher order moments of the signal. For the case where the permeability does affect the $D_{\mathbf{u}_{\mathbf{g}}}^{\text {eff }}$, the analysis is more difficult and we refer the reader to $[7,15,19,38,39]$ for results on periodic media and to $[3,8,30,31,32]$ on more general heterogeneous media, not necessarily periodic.

Now we summarize some existing results concerning the effective diffusion coefficient for finite domains where the membrane permeability is negligible. In the short time regime, the effective diffusion coefficient is reduced from the free diffusion coefficient $\mathcal{D}_{0}$ by the presence of the cell membranes that affects only the molecules in the adjacent layer. The thickness of this layer is of the order of the diffusion length $\sqrt{\mathcal{D}_{0} t}$ [17], where $\mathcal{D}_{0}$ is the bulk diffusion coefficient. Calculations in [26, 27] show

$$
D_{\text {short }}^{\mathrm{eff}}(t)=\mathcal{D}_{0}\left(1-\frac{4}{3 d \sqrt{\pi}} \frac{S}{V} \sqrt{\mathcal{D}_{0} t}\right),
$$


where $d$ is the spatial dimension and $S / V$ is the surface to volume ratio. This result was extended to include higher order terms accounting for permeable membranes, surface relaxation and mean curvature [18,30]. It was also shown that, in the case of anisotropic media subjected to a linear gradient with direction $\mathbf{u}_{\mathbf{g}}$, one should replace $\frac{S}{d V}$ above by $\frac{\int_{\partial \Omega}\left(\mathbf{u}_{\mathbf{g}} \cdot \nu\right)^{2} d \mathbf{x}}{|\Omega|}[1,9]$. In the long time limit, the spins explore the whole available space of the finite domain and then their mean square displacement saturates while the effective diffusion coefficient decreases as $\Delta$ increases. For an isolated cell of a typical size $R$ the diffusion becomes Gaussian as was shown in [29, 34]. In the case of the PGSE sequence in the narrow pulse limit one gets

$$
D_{\text {long }}^{\mathrm{eff}}(\Delta) \approx C \frac{R^{2}}{\Delta}
$$

where $C$ is a geometrical constant (for example, $C=1 / 4$ for the reflecting cylinder and $C=1 / 12$ for a $1 \mathrm{D}$ configuration $[4,9])$.

Finally, an approach that is closely related to the work of this paper is the "matrix formalism" approach used to describe restricted diffusion in bounded domains[2, $9,10,11]$. There one considers the applied diffusion-encoding magnetic field as a perturbation of the Laplace operator and the magnetization is decomposed on the basis of Laplacian eigenfunctions.

In contrast to the "matrix formalism" approach, the homogeneous model [14] which we call the H-ADC model and which is the focus of this paper, was derived using a certain scaling of the membrane permeability with respect to other physical parameters and thus is not limited to impermeable domains. Our derivation of the $\mathrm{H}-A D C$ model justifies neglecting the membrane permeability for the choice of scaling that we have made. In addition, since we have formulated the time-dependent effective diffusion coefficient as the solution of a diffusion equation rather than directly in the eigenfunction basis, we have the freedom to analyze the solution of the resulting diffusion equation using both the eigenfunction representation as well as the layer potential representation according the relevant time regime under consideration. Finally, we note that we preferred the term "apparent" to the term "effective" in naming the $\mathrm{H}-A D C$ model due to the more common usage of the term $A D C$ in the MRI community.

This paper is organized as follows. In Section 2 we describe the $\mathrm{H}-A D C$ model derived in [14]. In Section 3 we represent the solution of the relevant diffusion equation of the $\mathrm{H}-A D C$ model using the eigenfunction basis as well as by single layer potentials and discuss the regime where each representation is advantageous. In Section 4 we provide formulas for the effective diffusion coefficient that is averaged over diffusion-encoding gradient directions that are uniformly distributed in the unit sphere. In Section 5 we validate our analytical results with numerical simulations on two-dimensional geometries. Section 6 contains our conclusions.

2. Effective diffusion coefficient in finite domains. In a previous work [14], we obtained an homogenized model by starting from the Bloch-Torrey equation using the following scaling relationship between the time $(\Delta$ and $\delta)$, the biological cell membrane permeability $(\kappa)$, the diffusion-encoding magnetic field gradient strength $(g)$, and a periodicity length of the cellular geometry $(L)$ :

$$
L=O(\epsilon), \kappa=O(\epsilon), g=O\left(\epsilon^{-2}\right),\{\Delta, \delta\}=O\left(\epsilon^{2}\right),
$$

where $\epsilon$ is a non-dimensional parameter. It was shown that with this choice, there is no coupling between the different geometrical compartments in the $g^{2}$ term which 
gives rise to the effective diffusion coefficient. The total effective diffusion coefficient is the sum of the effective diffusion coefficient in each geometrical compartment weighted by its volume fraction. Thus, in this paper we are justified in considering each compartment separately.

According to [14], with the definitions of $F(t)$ given in (11), the effective diffusion coefficient in the compartment $\Omega$ can be obtained in the following way:

$$
D_{\mathbf{u}_{\mathbf{g}}}^{\mathrm{eff}}=\mathcal{D}_{0}-\frac{\mathcal{D}_{0}}{\int_{0}^{T E} F(t)^{2} d t} \int_{0}^{T E} F(t) h(t) d t,
$$

where

$$
h(t)=\frac{1}{|\Omega|} \int_{\Omega} \mathbf{u}_{\mathbf{g}} \cdot \nabla \omega(\mathbf{x}, t)
$$

is a quantity related to the directional gradient of a function $\omega$ that is the solution of the homogeneous diffusion equation with Neumann boundary condition and zero initial condition:

$$
\begin{aligned}
\frac{\partial}{\partial t} \omega(\mathbf{x}, t)-\nabla\left(\mathcal{D}_{0} \nabla \omega(\mathbf{x}, t)\right) & =0, & \mathbf{x} \in \Omega, \\
\mathcal{D}_{0} \nabla \omega(\mathbf{x}, t) \cdot \nu(\mathbf{x}) & =\mathcal{D}_{0} F(t) \mathbf{u}_{\mathbf{g}} \cdot \nu(\mathbf{x}), & \mathbf{x} \in \partial \Omega, \\
\omega(\mathbf{x}, t) & =0, & \mathbf{x} \in \Omega,
\end{aligned}
$$

$\nu$ is the outward normal and $t \in[0, T E]$. We can see that if $h(t)$ is close to $F(t)$, then $D_{\mathbf{u}_{\mathbf{g}}}^{\text {eff }}$ is close to 0 . The above set of equations, (15)-(17), comprise the homogenized model that we call the $\mathrm{H}-A D C$ model.

In our previous work [14], we imposed periodic boundary conditions on the boundary of the voxel. In this paper, we are interested in analyzing (15)-(17) for spatially finite compartments, which is relevant to diffusion inside biological cells. It will not be necessary to impose periodic boundary conditions on the sides of the imaging voxel if we consider only cells that do not touch the sides.

3. Solution of the model. Defining the right hand side of the Neumann boundary condition as

$$
\beta(\mathbf{y}, t):=\mathcal{D}_{0} F(t) \mathbf{u}_{\mathbf{g}} \cdot \nu(\mathbf{y}),
$$

we will use the following two equivalent expressions for $h(t)$ :

$$
h(t)=\frac{1}{|\Omega|} \int_{\Omega} \mathbf{u}_{\mathbf{g}} \cdot \nabla \omega(\mathbf{x}, t) d \mathbf{x}=\frac{1}{|\Omega|} \int_{\Gamma} \omega(\mathbf{y}, t)\left(\mathbf{u}_{\mathbf{g}} \cdot \nu(\mathbf{y})\right) d s_{\mathbf{y}},
$$

where the second expression can be obtained by applying the divergence theorem to (16). We observe that the first expression uses values of the gradient of $\omega$ inside the domain while the second uses the values of $\omega$ on the boundary. Each expression will have advantages depending on whether we use the eigenfunctions of the Laplace operator or layer potentials to represent $\omega$.

3.1. Eigenfunctions representation. Writing $\omega$ as the sum

$$
\omega(\mathbf{x}, t)=\widetilde{\omega}(\mathbf{x}, t)+F(t) \mathbf{x} \cdot \mathbf{u}_{\mathbf{g}}, \mathbf{x} \in \Omega, t \in[0, T E]
$$


where $\widetilde{\omega}(\mathbf{x}, t)$ satisfied the diffusion equation with a forcing term and homogeneous boundary condition:

$$
\begin{array}{rlrl}
\frac{\partial}{\partial t} \widetilde{\omega}(\mathbf{x}, t)-\nabla\left(\mathcal{D}_{0} \nabla \widetilde{\omega}(\mathbf{x}, t)\right) & =-f(t) \mathbf{x} \cdot \mathbf{u}_{\mathbf{g}}, & \mathbf{x} \in \Omega, t \in[0, T E], \\
\mathcal{D}_{0} \nabla \widetilde{\omega}(\mathbf{x}, t) \cdot \nu(\mathbf{x}) & =0, & \mathbf{x} \in \Gamma, t \in[0, T E], \\
\widetilde{\omega}(\mathbf{x}, t) & =0, & & \mathbf{x} \in \Omega,
\end{array}
$$

it is well-known that $\widetilde{\omega}(\mathbf{x}, t)$ can be expanded in the basis of Laplace eigenfunctions. Let $\phi_{n}(\mathbf{x})$ and $\lambda_{n}$ be the $L^{2}$ normalized eigenfunctions and eigenvalues associated to the Laplace operator with homogeneous Neumann boundary conditions:

$$
\begin{array}{llrl}
-\nabla \mathcal{D}_{0}\left(\nabla \phi_{n}(\mathbf{x})\right) & =\lambda_{n} \phi_{n}(\mathbf{x}), & & \mathbf{x} \in \Omega, \\
\mathcal{D}_{0} \nabla \phi_{n}(\mathbf{x}) \cdot \nu(\mathbf{x}) & =0, & & \mathbf{x} \in \Gamma .
\end{array}
$$

We can write $\widetilde{\omega}(\mathbf{x}, t)$ in the basis of the eigenfunctions as

$$
\widetilde{\omega}(\mathbf{x}, t)=-a_{0} \phi_{0}(\mathbf{x}) F(t)+\sum_{n=1}^{\infty}\left(-a_{n}\right) \phi_{n}(\mathbf{x}) \int_{0}^{t} e^{-\mathcal{D}_{0} \lambda_{n}(t-s)} f(s) d s
$$

where the coefficients are

$$
a_{0}=\frac{1}{\sqrt{|\Omega|}} \int_{\Omega} \mathbf{x} \cdot \mathbf{u}_{\mathbf{g}} d \mathbf{x}, \quad a_{n}=\int_{\Omega} \mathbf{x} \cdot \mathbf{u}_{\mathbf{g}} \phi_{n}(\mathbf{x}) d \mathbf{x},
$$

which coincide with the first moments of the eigenfunctions in the $\mathbf{u}_{\mathrm{g}}$ direction.

Finally, the solution to the diffusion equation is

$$
\omega(\mathbf{x}, t)=\left(\mathbf{x} \cdot \mathbf{u}_{\mathbf{g}}-\frac{1}{\sqrt{|\Omega|}} a_{0}\right) F(t)+\sum_{n=1}^{\infty}\left(-a_{n}\right) \phi_{n}(\mathbf{x}) \int_{0}^{t} e^{-\mathcal{D}_{0} \lambda_{n}(t-s)} f(s) d s
$$

and using properties of the eigenfunctions:

$$
\int_{\Omega} \phi_{n}(\mathbf{x}) d \mathbf{x}= \begin{cases}\sqrt{|\Omega|}, & n=0 \\ 0, & n \geq 1,\end{cases}
$$

and the divergence theorem:

(28)

$\int_{\Omega} \lambda_{n} \phi_{n}\left(\mathbf{u}_{\mathbf{g}} \cdot \mathbf{x}\right) d \mathbf{x}=\int_{\Omega} \nabla \phi_{n}(\mathbf{x}) \cdot \mathbf{u}_{\mathbf{g}} d \mathbf{x}-\int_{\Gamma} \mathcal{D}_{0} \nabla \phi_{n}(\mathbf{x}) \cdot \nu \mathbf{u}_{\mathbf{g}} \cdot \nu d s_{\mathbf{x}}=\int_{\Omega} \nabla \phi_{n}(\mathbf{x}) \cdot \mathbf{u}_{\mathbf{g}} d \mathbf{x}$

we obtain

$$
h(t)=F(t)+\sum_{n=1}^{\infty}-\frac{\left(a_{n}\right)^{2} \lambda_{n}}{|\Omega|} \int_{0}^{t} e^{-\mathcal{D}_{0} \lambda_{n}(t-s)} f(s) d s .
$$

This leads to the final formula:

$$
D_{\mathbf{u}_{\mathbf{g}}}^{\mathrm{eff}}=\sum_{n=1}^{\infty} \frac{\left(a_{n}\right)^{2} \mathcal{D}_{0} \lambda_{n}}{|\Omega| \int_{0}^{T E} F^{2}(t) d t} \int_{0}^{T E} F(t)\left(\int_{0}^{t} e^{-\mathcal{D}_{0} \lambda_{n}(t-s)} f(s) d s\right) d t .
$$


We remark that this formula is the same as the one obtained with the matrix formalism in [9]. In particular, if we consider PGSE sequence, we can rewrite (15) using the contribution of each of the three intervals as

$(31)$

$$
D_{\mathbf{u}_{\mathrm{g}}}^{\mathrm{eff}}=\mathcal{D}_{0}-(\underbrace{\frac{\mathcal{D}_{0}}{A} \int_{0}^{\delta} t h(t) d t}_{I}+\underbrace{\frac{\mathcal{D}_{0}}{A} \int_{\delta}^{\Delta} \delta h(t) d t}_{I I}+\underbrace{\frac{\mathcal{D}_{0}}{A} \int_{\Delta}^{\Delta+\delta}(\Delta+\delta-t) h(t) d t}_{I I I}),
$$

where

$$
A=\int_{0}^{T E} F^{2}(t) d t=\delta^{2}\left(\Delta-\frac{\delta}{3}\right)
$$

and in the first pulse

$$
I=\frac{\mathcal{D}_{0} \delta^{3}}{3 A}+\frac{1}{|\Omega| A} \sum_{n=1}^{\infty}\left(a_{n}\right)^{2}\left(-\frac{\delta^{2}}{2}-\frac{\delta e^{-\mathcal{D}_{0} \lambda_{n} \delta}}{\mathcal{D}_{0} \lambda_{n}}-\frac{e^{-\mathcal{D}_{0} \lambda_{n} \delta}-1}{\left(\mathcal{D}_{0} \lambda_{n}\right)^{2}}\right),
$$

between the pulses

$$
I I=\frac{\mathcal{D}_{0} \delta^{2}(\Delta-\delta)}{A}+\frac{1}{|\Omega| A} \sum_{n=1}^{\infty} \frac{-\delta\left(a_{n}\right)^{2}}{\mathcal{D}_{0} \lambda_{n}}\left(e^{-\mathcal{D}_{0} \lambda_{n} \Delta}-e^{-\mathcal{D}_{0} \lambda_{n}(\Delta-\delta)}-e^{-\mathcal{D}_{0} \lambda_{n} \delta}+1\right),
$$

and in the second pulse

$$
\begin{aligned}
I I I=\frac{\mathcal{D}_{0} \delta^{3}}{3 A}+ & \sum_{n=1}^{\infty} \frac{-\left(a_{n}\right)^{2}}{\mathcal{D}_{0} \lambda_{n} A|\Omega|}\left(\delta-\frac{\delta^{2} \mathcal{D}_{0} \lambda_{n}}{2}-\delta e^{-\mathcal{D}_{0} \lambda_{n} \Delta}+\delta e^{-\mathcal{D}_{0} \lambda_{n}(\Delta-\delta)}\right. \\
& \left.+\frac{2 e^{-\mathcal{D}_{0} \lambda_{n} \Delta}-1+e^{-\mathcal{D}_{0} \lambda_{n} \delta}-e^{-\mathcal{D}_{0} \lambda_{n}(\Delta+\delta)}-e^{-\mathcal{D}_{0} \lambda_{n}(\Delta-\delta)}}{\mathcal{D}_{0} \lambda_{n}}\right)
\end{aligned}
$$

In the end, we obtain

$$
\begin{aligned}
D_{\mathbf{u}_{\mathbf{g}}}^{\mathrm{eff}}=\sum_{n=1}^{\infty} \frac{-\left(a_{n}\right)^{2}}{\mathcal{D}_{0}^{2} \lambda_{n}^{2} \delta^{2}\left(\Delta-\frac{\delta}{3}\right)|\Omega|}\left[e^{-\mathcal{D}_{0} \lambda_{n}(\Delta+\delta)}+e^{-\mathcal{D}_{0} \lambda_{n}(\Delta-\delta)}\right. & \\
& \left.-2\left(\mathcal{D}_{0} \lambda_{n} \delta+e^{-\mathcal{D}_{0} \lambda_{n} \delta}+e^{-\mathcal{D}_{0} \lambda_{n} \Delta}-1\right)\right]
\end{aligned}
$$

In the narrow pulse case $(\delta \ll \Delta)$, we obtain

$$
D_{\mathbf{u}_{\mathrm{g}}}^{\mathrm{eff}} \approx \sum_{n=1}^{\infty} \frac{\left(a_{n}\right)^{2}}{\Delta}\left(1-e^{-\mathcal{D}_{0} \lambda_{n} \Delta}\right)
$$

which confirms that $D_{\mathbf{u}_{\mathbf{g}}}^{\text {eff }}$ approach its long time limit as proportional to $1 / \Delta$ inside finite domains. In particular, for a $1 \mathrm{D}$ configuration of length $L, a_{1}=\frac{L^{2}}{12}$ and for a reflecting cylinder of radius $R, a_{1}=\frac{R^{2}}{4}$, which confirm the results in $[4,9]$. 
3.2. Layer potential representation. The solution of the diffusion equation can be also represented using layer potentials [13]. This representation is more efficient than the eigenfunction representation at short diffusion times. Since the PDE has a Neumann boundary condition, we choose to represent the solution $\omega(\mathbf{x}, t)=S[\mu](\mathbf{x}, t)$ as a single layer potential with a density $\mu$ defined on $\Gamma$,

$$
S[\mu](\mathbf{x}, t)=\int_{0}^{t} \int_{\Gamma} \mathcal{D}_{0} G(\mathbf{x}-\mathbf{y}, t-\tau) \mu(\mathbf{y}, \tau) d s_{\mathbf{y}} d \tau,
$$

where $G(\mathbf{x}, t)$ is the fundamental solution of the heat equation in free space given by

$$
G(\mathbf{x}, t)=\left(4 \pi \mathcal{D}_{0} t\right)^{-d / 2} \exp \left(\frac{-\|\mathbf{x}\|^{2}}{4 \mathcal{D}_{0} t}\right)
$$

and $d$ is the space dimension. At short times, there is an unavoidable square root singulary in $t$ in the single layer potential, therefore in what follows, we separate out the integrand in (38) in the following way,

$$
S[\mu](\mathbf{x}, t)=\int_{0}^{t} \frac{1}{\sqrt{4 \mathcal{D}_{0} \pi(t-\tau)}} B_{S}[\mu](\mathbf{x}, t, \tau) d \tau,
$$

where

$$
B_{S}[\mu](\mathbf{x}, t, \tau):=\int_{\Gamma} \mathcal{D}_{0} \sqrt{4 \mathcal{D}_{0} \pi(t-\tau)} G(\mathbf{x}-\mathbf{y}, t-\tau) \mu(\mathbf{y}, \tau) d s_{\mathbf{y}}
$$

is analytic in time if $\mu$ is. The single layer potential satisfies

$$
\begin{aligned}
\frac{\partial}{\partial t} S[\mu](\mathbf{x}, t)-\nabla\left(\mathcal{D}_{0} \nabla S[\mu](\mathbf{x}, t)\right) & =0, & \mathbf{x} \in \Omega, t \in[0, T E], \\
S[\mu](\mathbf{x}, 0) & =0, & \mathbf{x} \in \Omega .
\end{aligned}
$$

Then the density $\mu$ is chosen to be a causal function and is determined by imposing the Neumann boundary conditions:

$$
\lim _{\mathbf{x} \rightarrow \mathbf{x}^{0} \in \Gamma} \mathcal{D}_{0} \nabla S[\mu](\mathbf{x}, t) \cdot \nu(\mathbf{x})=\beta\left(\mathbf{x}^{0}, t\right), \quad \mathbf{x}^{0} \in \Gamma, t \in[0, T E],
$$

where $\beta\left(\mathbf{x}^{0}, t\right)$ is defined in (18). Using the jump properties of the traces of double layer potentials, the integral equation to be solved for $\mu$ is then the following:

$$
\frac{\mathcal{D}_{0}}{2} \mu\left(\mathbf{x}^{0}, t\right)+\mathcal{D}_{0} K^{*}[\mu]\left(\mathbf{x}^{0}, t\right)=\beta\left(\mathbf{x}^{0}, t\right), \quad \mathbf{x}^{0} \in \Gamma, t \in[0, T E],
$$

where

$$
K^{*}[\mu]\left(\mathbf{x}^{0}, t\right)=\int_{0}^{t} \frac{1}{\sqrt{4 \pi \mathcal{D}_{0}(t-\tau)}} B_{K}[\mu]\left(\mathbf{x}^{0}, t, \tau\right) d \tau
$$

with

$$
\begin{aligned}
& B_{K}[\mu]\left(\mathbf{x}^{0}, t, \tau\right) \equiv \\
& \int_{\Gamma} \frac{-2\left(\mathbf{x}^{0}-\mathbf{y}\right) \cdot \nu(\mathbf{y})}{4 \mathcal{D}_{0}(t-\tau)} \mathcal{D}_{0} \sqrt{4 \pi \mathcal{D}_{0}(t-\tau)} G\left(\mathbf{x}^{0}-\mathbf{y}, t-\tau\right) \mu(\mathbf{y}, \tau) d s_{\mathbf{y}}
\end{aligned}
$$


being analytic in time if $\mu$ is.

To compute $h(t)$ from (19) we only need to evaluate $\omega\left(\mathbf{x}^{0}, t\right)=S[\mu]\left(\mathbf{x}^{0}, t\right)$ on the boundary $\Gamma$. We write the density $\mu$ as the solution of (44):

$$
\mu\left(\mathbf{x}^{0}, t\right)=\frac{2}{\mathcal{D}_{0}}\left(1+2 K^{*}\right)^{-1} \beta\left(\mathbf{x}^{0}, t\right), \quad \mathbf{x}^{0} \in \Gamma, t \in[0, T E],
$$

and expand the operator $\left(1+2 K^{*}\right)^{-1}$ for short time $t$ (corresponding with $2 K^{*}$ being a contraction) as

$$
\mu\left(\mathbf{x}^{0}, t\right)=\frac{2}{\mathcal{D}_{0}}\left(1-2 K^{*}+4\left(K^{*}\right)^{2}+\ldots\right) \beta\left(\mathbf{x}^{0}, t\right),
$$

to obtain

$$
\mu\left(\mathbf{x}^{0}, t\right)=\frac{2}{\mathcal{D}_{0}} \beta\left(\mathbf{x}^{0}, t\right)-\frac{4}{\mathcal{D}_{0}} K^{*}[\beta]\left(\mathbf{x}^{0}, t\right)+\text { higher order terms. }
$$

This means in particular that

$$
\begin{aligned}
S[\mu]\left(\mathbf{x}^{0}, t\right)=S\left[\frac{2}{\mathcal{D}_{0}} \beta\right]\left(\mathbf{x}^{0}, t\right) & +S\left[-\frac{4}{\mathcal{D}_{0}} K^{*}[\beta]\right]\left(\mathbf{x}^{0}, t\right) \\
& + \text { higher order terms. }
\end{aligned}
$$

We will now compute the first term on the right hand side of the above equation to get an approximate expression for $\omega\left(\mathbf{x}^{0}, t\right)=S[\mu]\left(\mathbf{x}^{0}, t\right)$ and we will compute the second term to get an expression for the error.

We note here that asymptotic results for small values of $(t-\tau)$ concerning $B_{S}[\mu]$ and $B_{K}[\mu]$ for a density $\mu$ that has a Taylor expansion in both the space and the time variables have been obtained in $[12,24]$, but only in two dimensions. However, we expect that the generalization to three dimensions should be straightforward. In particular, the curvature term and higher order spatial derivatives need to be generalized to analogous quantities in three dimensions.

Here, we summarize the results derived in $[12,24]$ for two dimensions. Suppose a local parametrization for $\Gamma$ around $\mathbf{x}^{0}$ of the form

$$
\Gamma=\{(s, y(s)), s=-\infty \cdots \infty\},
$$

having translated and rotated the $x$ and $y$-axes so that the origin is at $\mathbf{x}^{0}$ and the tangent of $\Gamma$ at $\mathbf{x}^{0}$ is parallel to the $x$-axis, with the end points extended to $\pm \infty$ because such an approximation facilitates the computation of the integrals and the resulting error is exponentially small. Let $y(s)$ be oriented counter-clockwise, with the following expansion in terms of the curvilinear abscissa $s$ :

$$
y(s)=\frac{1}{2} \gamma_{s s} s^{2}+\frac{1}{6} \gamma_{s s s} s^{3}+\frac{1}{24} \gamma_{s s s s} s^{4}+\ldots
$$

For the operator $S$, it was shown in $[12,24]$ that:

$$
\begin{aligned}
B_{S}[\mu]\left(\mathbf{x}^{0}, t, \tau\right) & =\mu\left(\mathbf{x}^{0}, t\right)+\left(\frac{\gamma_{s s}\left(\mathbf{x}^{0}\right)^{2}}{4} \mu\left(\mathbf{x}^{0}, t\right)+\mu_{t}\left(\mathbf{x}^{0}, t\right)-\mu_{s s}\left(\mathbf{x}^{0}, t\right)\right)(t-\tau) \\
& +O\left((t-\tau)^{2}\right)
\end{aligned}
$$


and for the operator $K^{*}$ it was shown that:

$$
\begin{aligned}
B_{K}[\mu]\left(\mathbf{x}^{0}, t, \tau\right) & =\frac{\gamma_{s s}\left(\mathbf{x}^{0}\right)}{2} \mu\left(\mathbf{x}^{0}, t\right)+\frac{1}{8}\left[16 \gamma_{s s s}\left(\mathbf{x}^{0}\right) \mu_{s}\left(\mathbf{x}^{0}, t\right)+12 \gamma_{s s}\left(\mathbf{x}^{0}\right) \mu_{s s}\left(\mathbf{x}^{0}, t\right)\right. \\
& \left.+4 \gamma_{s s}\left(\mathbf{x}^{0}\right) \mu_{t}\left(\mathbf{x}^{0}, t\right)+\left(6 \gamma_{s s s s}\left(\mathbf{x}^{0}\right)-15 \gamma_{s s}\left(\mathbf{x}^{0}\right)^{3}\right) \mu\left(\mathbf{x}^{0}, t\right)\right](t-\tau) \\
& +O\left((t-\tau)^{2}\right)
\end{aligned}
$$

Specifically $\gamma_{s s}$ is the curvature of $\Gamma$ at the point $\mathbf{x}^{0}$ and in what follows we will indicate it as $k\left(\mathbf{x}^{0}\right)$. In three dimensions, it should be easy to see that the constant term in $B_{S}[\mu]\left(\mathbf{x}^{0}, t, \tau\right)$ would not change, and the constant term in $B_{K}[\mu]\left(\mathbf{x}^{0}, t, \tau\right)$ would contain spatial derivatives on a two dimensional manifold.

For the PGSE sequence, $\beta\left(\mathbf{x}^{0}, t\right)$ assumes the following three expressions in the three time intervals:

$$
\beta\left(\mathbf{x}^{0}, t\right)=\mathcal{D}_{0} \mathbf{u}_{\mathbf{g}} \cdot \nu\left(\mathbf{x}^{0}\right) \begin{cases}t & 0<t \leq \delta, \\ \delta & \delta<t \leq \Delta, \\ \Delta+\delta-t & \Delta<t \leq \Delta+\delta .\end{cases}
$$

First, using the definition (40) and the result (52) we obtain

$$
\begin{aligned}
& S\left[\frac{2}{\mathcal{D}_{0}} \beta\right]\left(\mathbf{x}^{0}, t\right)=\frac{4\left(\mathcal{D}_{0}\right)^{1 / 2}}{3 \sqrt{\pi}} \mathbf{u}_{\mathbf{g}} \cdot \nu\left(\mathbf{x}^{0}\right)\left\{\begin{array}{l}
t^{3 / 2} \\
t^{3 / 2}-(t-\delta)^{3 / 2} \\
t^{3 / 2}-(t-\delta)^{3 / 2}-(t-\Delta)^{3 / 2}
\end{array}\right. \\
& +O \begin{cases}\left(t^{5 / 2}\right) & \text { if } 0<t \leq \delta, \\
\left(t^{5 / 2}-(t-\delta)^{5 / 2}\right) & \text { if } \delta<t \leq \Delta, \\
\left(t^{5 / 2}-(t-\delta)^{5 / 2}-(t-\Delta)^{5 / 2}\right) & \text { if } \Delta<t \leq \Delta+\delta .\end{cases}
\end{aligned}
$$

Similarly, using the definition (45) and the result (53) we obtain

$$
K^{*}[\beta]\left(\mathbf{x}^{0}, t\right)=\alpha_{1}\left(\mathbf{x}_{0}\right) \alpha_{2}(t)+O\left(\alpha_{3}(t)\right)
$$

where

$$
\begin{aligned}
& \alpha_{1}\left(\mathbf{x}_{0}\right)= \frac{\left(\mathcal{D}_{0}\right)^{3 / 2} k\left(\mathbf{x}^{0}\right)}{3 \sqrt{\pi}}\left(\mathbf{u}_{\mathbf{g}} \cdot \nu\left(\mathbf{x}^{0}\right)\right), \\
& \alpha_{2}(t)= \begin{cases}t^{3 / 2} & \text { if } 0<t \leq \delta, \\
t^{3 / 2}-(t-\delta)^{3 / 2} & \text { if } \delta<t \leq \Delta, \\
t^{3 / 2}-(t-\delta)^{3 / 2}-(t-\Delta)^{3 / 2} & \text { if } \Delta<t \leq \Delta+\delta,\end{cases} \\
& \alpha_{3}(t)= \begin{cases}t^{5 / 2} & \text { if } 0<t \leq \delta, \\
t^{5 / 2}-(t-\delta)^{5 / 2} & \text { if } \delta<t \leq \Delta, \\
t^{5 / 2}-(t-\delta)^{5 / 2}-(t-\Delta)^{5 / 2} & \text { if } \Delta<t \leq \Delta+\delta .\end{cases}
\end{aligned}
$$

To compute $S\left[-\frac{4}{\mathcal{D}_{0}} K^{*}[\beta]\right]\left(\mathbf{x}^{0}, t\right)$, we first observe that $S\left[-\frac{4}{\mathcal{D}_{0}} K^{*}[\beta]\right]\left(\mathbf{x}^{0}, t\right)=-\frac{4}{\mathcal{D}_{0}} S\left[K^{*}[\beta]\right]\left(\mathbf{x}^{0}, t\right)$. Moreover, to compute $S\left[K^{*}[\beta]\right]\left(\mathbf{x}^{0}, t\right)$ we cannot use the result $(52)$ because $\alpha_{2}$ and 
$\alpha_{3}$ do not have a Taylor expansion in $t$. Following the idea in [12], we explicitly compute the lowest order terms of $S\left[K^{*}[\beta]\right]\left(\mathbf{x}^{0}, t\right)$ in two dimensions by

$$
S\left[K^{*}[\beta]\right]\left(\mathbf{x}_{0}, t\right)=\int_{0}^{t} \frac{1}{\sqrt{4 \pi \mathcal{D}_{0}(t-\tau)}} B_{S}\left[K^{*}[\beta]\right]\left(\mathbf{x}_{0}, t, \tau\right) d \tau,
$$

where

$$
\begin{aligned}
& B_{S}\left[K^{*}[\beta]\right]\left(\mathbf{x}_{0}, t, \tau\right) \\
& =\int_{-\infty}^{+\infty} \mathcal{D}_{0} \frac{e^{-\frac{s^{2}+y(s)^{2}}{4 \mathcal{D}_{0}(t-\tau)}}}{\sqrt{4 \pi \mathcal{D}_{0}(t-\tau)}}\left(\alpha_{1}(s) \alpha_{2}(\tau)+O\left(\alpha_{3}(\tau)\right)\right) \sqrt{1+\left(y^{\prime}(s)\right)^{2}} d s .
\end{aligned}
$$

Note for simplicity, we replaced $\alpha_{1}\left(\mathbf{x}_{0}\right)$ by $\alpha_{1}(s)$ to indicate the local parametrization of $\Gamma$ around $\mathbf{x}_{0}$, as described previously.

To compute the above spatial integral we note the dominant contribution of the Gaussian $e^{-\frac{s^{2}}{4 \mathcal{D}_{0}(t-\tau)}}$ and make the change of variables $r=\frac{s}{\sqrt{4 \mathcal{D}_{0}(t-\tau)}}$ to obtain

$$
B_{S}\left[K^{*}[\beta]\right]\left(\mathbf{x}_{0}, t, \tau\right)=\int_{-\infty}^{+\infty} \frac{\mathcal{D}_{0}}{\sqrt{\pi}} e^{-r^{2}} e^{-\frac{y(r v)^{2}}{v^{2}}}
$$

$$
\left(\alpha_{1}(r v) \alpha_{2}\left(t-\frac{v^{2}}{4 \mathcal{D}_{0}}\right)+O\left(\alpha_{3}\left(t-\frac{v^{2}}{4 \mathcal{D}_{0}}\right)\right)\right) \sqrt{1+\left(y^{\prime}(r v)\right)^{2}} d r
$$

where $v=\sqrt{4 \mathcal{D}_{0}(t-\tau)}$. We would like an asymptotic expansion of the above integral in $v$. We note $y(r v)=\frac{1}{2} k\left(\mathbf{x}_{0}\right) r^{2} v^{2}+O\left(r^{3} v^{3}\right)$ and $\alpha_{1}(0)=\frac{\left(\mathcal{D}_{0}\right)^{3 / 2} k\left(\mathbf{x}^{0}\right)}{3 \sqrt{\pi}}\left(\mathbf{u}_{\mathbf{g}} \cdot \nu\left(\mathbf{x}^{0}\right)\right)$. The order $O(v)$ term only occurs in $\alpha_{1}$ and we do not need to take it into account due to the anti-symmetry of $e^{-r^{2}} r$. So we compute the space integral to obtain

$$
\begin{aligned}
& =\int_{-\infty}^{+\infty} \frac{\mathcal{D}_{0}}{\sqrt{\pi}} e^{-r^{2}}\left(\left(\alpha_{1}(0)+O\left(r^{2} v^{2}\right)\right) \alpha_{2}\left(t-\frac{v^{2}}{4 \mathcal{D}_{0}}\right)+O\left(\alpha_{3}\left(t-\frac{v^{2}}{4 \mathcal{D}_{0}}\right)\right)\right) d r \\
& =\alpha_{2}\left(t-\frac{v^{2}}{4 \mathcal{D}_{0}}\right)\left(\mathcal{D}_{0} \alpha_{1}(0)+O\left(v^{2}\right)\right)+O\left(\alpha_{3}\left(t-\frac{v^{2}}{4 \mathcal{D}_{0}}\right)\right) .
\end{aligned}
$$

We now take the above expression and put it into the time integral to get $S\left[K^{*}[\beta]\right]\left(\mathbf{x}_{0}, t\right):$

$$
\int_{0}^{t} \frac{1}{\sqrt{4 \pi \mathcal{D}_{0}(t-\tau)}}\left(\alpha_{2}(\tau)\left(\mathcal{D}_{0} \alpha_{1}(0)+O(t-\tau)\right)+O\left(\alpha_{3}(\tau)\right)\right) d \tau .
$$

Using the following property of the beta function (the Euler integral of the first kind)

$$
\int_{0}^{t}(t-\tau)^{w} \tau^{p} d \tau=t^{p+w+1} \frac{\Gamma(p+1) \Gamma(w+1)}{\Gamma(w+p+2)},
$$


368 we can compute $\int_{0}^{t} \frac{1}{\sqrt{(t-\tau)}} \alpha_{2}(\tau) d \tau$ exactly:

369

$(60)$

$$
\begin{aligned}
& \int_{0}^{t} \frac{\tau^{\frac{3}{2}}}{\sqrt{t-\tau}} d \tau=\frac{3 \pi}{8} t^{2} \\
& \int_{0}^{\delta} \frac{\tau^{\frac{3}{2}}}{\sqrt{t-\tau}} d \tau+\int_{\delta}^{t} \frac{\tau^{\frac{3}{2}}-(\tau-\delta)^{\frac{3}{2}}}{\sqrt{t-\tau}} d \tau=\frac{3 \pi}{8}\left(t^{2}-(t-\delta)^{2}\right) \\
& \int_{0}^{\delta} \frac{\tau^{\frac{3}{2}}}{\sqrt{t-\tau}} d \tau+\int_{\delta}^{\Delta} \frac{\tau^{\frac{3}{2}}-(\tau-\delta)^{\frac{3}{2}}}{\sqrt{t-\tau}} d \tau+\int_{\Delta}^{t} \frac{\tau^{\frac{3}{2}}-(\tau-\delta)^{\frac{3}{2}}-(\tau-\Delta)^{\frac{3}{2}}}{\sqrt{t-\tau}} d \tau \\
& =\frac{3 \pi}{8}\left(t^{2}-(t-\delta)^{2}-(t-\Delta)^{2}\right) .
\end{aligned}
$$

Therefore, the dominant asymptotic terms are:

(61)

371

$$
\begin{aligned}
& S\left[-\frac{4}{\mathcal{D}_{0}} K^{*}[\beta]\right]\left(\mathbf{x}^{0}, t\right)=-\frac{4}{\mathcal{D}_{0}} \frac{\mathcal{D}_{0}^{2}}{16} k\left(\mathbf{x}^{0}\right)\left(\mathbf{u}_{\mathbf{g}} \cdot \nu\left(\mathbf{x}^{0}\right)\right)\left\{\begin{array}{l}
t^{2} \\
t^{2}-(t-\delta)^{2} \\
t^{2}-(t-\delta)^{2}-(t-\Delta)^{2} .
\end{array}\right. \\
& +O \begin{cases}\left(t^{3}\right) & \text { if } 0<t \leq \delta, \\
\left(t^{3}-(t-\delta)^{3}\right), & \text { if } \delta<t \leq \Delta, \\
\left(t^{3}-(t-\delta)^{3}-(t-\Delta)^{3}\right) & \text { if } \Delta<t \leq \Delta+\delta,\end{cases}
\end{aligned}
$$

372 where we computed the error term by evaluating $\int_{0}^{t} \frac{1}{\sqrt{(t-\tau)}} \alpha_{2}(\tau)(t-\tau) d \tau$ and $373 \int_{0}^{t} \frac{1}{\sqrt{(t-\tau)}} \alpha_{3}(\tau) d \tau$, again using property of the beta function. Namely, for $374 \int_{0}^{t} \frac{1}{\sqrt{(t-\tau)}} \alpha_{2}(\tau)(t-\tau) d \tau$, we have

$$
\begin{aligned}
& \int_{0}^{t} \frac{\tau^{\frac{3}{2}}}{\sqrt{t-\tau}}(t-\tau) d \tau=\frac{\pi}{16} t^{3}, \\
& \int_{0}^{\delta} \frac{\tau^{\frac{3}{2}}}{\sqrt{t-\tau}}(t-\tau) d \tau+\int_{\delta}^{t} \frac{\tau^{\frac{3}{2}}-(\tau-\delta)^{\frac{3}{2}}}{\sqrt{t-\tau}}(t-\tau) d \tau=\frac{\pi}{16}\left(t^{3}-(t-\delta)^{3}\right) \\
& \int_{0}^{\delta} \frac{\tau^{\frac{3}{2}}}{\sqrt{t-\tau}}(t-\tau) d \tau+\int_{\delta}^{\Delta} \frac{\tau^{\frac{3}{2}}-(\tau-\delta)^{\frac{3}{2}}}{\sqrt{t-\tau}}(t-\tau) d \tau \\
& +\int_{\Delta}^{t} \frac{\tau^{\frac{3}{2}}-(\tau-\delta)^{\frac{3}{2}}-(\tau-\Delta)^{\frac{3}{2}}}{\sqrt{t-\tau}}(t-\tau) d \tau \\
& =\frac{\pi}{16}\left(t^{3}-(t-\delta)^{3}-(t-\Delta)^{3}\right) .
\end{aligned}
$$


For $\int_{0}^{t} \frac{1}{\sqrt{(t-\tau)}} \alpha_{3}(\tau) d \tau$, we have

$$
\begin{aligned}
& \int_{0}^{t} \frac{\tau^{\frac{5}{2}}}{\sqrt{t-\tau}} d \tau=\frac{5 \pi}{16} t^{3} \\
& \int_{0}^{\delta} \frac{\tau^{\frac{5}{2}}}{\sqrt{t-\tau}} d \tau+\int_{\delta}^{t} \frac{\tau^{\frac{5}{2}}-(\tau-\delta)^{\frac{3}{2}}}{\sqrt{t-\tau}} d \tau=\frac{5 \pi}{16}\left(t^{3}-(t-\delta)^{3}\right) \\
& \int_{0}^{\delta} \frac{\tau^{\frac{5}{2}}}{\sqrt{t-\tau}} d \tau+\int_{\delta}^{\Delta} \frac{\tau^{\frac{5}{2}}-(\tau-\delta)^{\frac{5}{2}}}{\sqrt{t-\tau}} d \tau+\int_{\Delta}^{t} \frac{\tau^{\frac{5}{2}}-(\tau-\delta)^{\frac{5}{2}}-(\tau-\Delta)^{\frac{5}{2}}}{\sqrt{t-\tau}} d \tau \\
& =\frac{5 \pi}{16}\left(t^{3}-(t-\delta)^{3}-(t-\Delta)^{3}\right) .
\end{aligned}
$$

Replacing the various expressions in (50) with the calculations we did above, we obtain the approximation with the error bound:

$(64)$

$$
S[\mu]\left(\mathbf{x}^{0}, t\right)=\frac{4\left(\mathcal{D}_{0}\right)^{1 / 2}}{3 \sqrt{\pi}} \mathbf{u}_{\mathbf{g}} \cdot \nu\left(\mathbf{x}^{0}\right)\left\{\begin{array}{l}
t^{3 / 2} \\
t^{3 / 2}-(t-\delta)^{3 / 2} \\
t^{3 / 2}-(t-\delta)^{3 / 2}-(t-\Delta)^{3 / 2}
\end{array}\right.
$$

$$
-\frac{\mathcal{D}_{0}}{16} k\left(\mathbf{x}^{0}\right)\left(\mathbf{u}_{\mathbf{g}} \cdot \nu\left(\mathbf{x}^{0}\right)\right)\left\{\begin{array}{l}
t^{2} \\
t^{2}-(t-\delta)^{2} \\
t^{2}-(t-\delta)^{2}-(t-\Delta)^{2}
\end{array} \quad+\right.\text { higher order terms. }
$$

Now using (64) we compute the approximate expressions of $h(t)$ in each time-interval with the corresponding errors in time.

In the first interval, we obtain

$$
h(t)=\frac{1}{|\Omega|} \int_{\Gamma} \omega(\mathbf{x}, t)\left(\mathbf{u}_{\mathbf{g}} \cdot \nu(\mathbf{x})\right) d s_{\mathbf{x}}=P t^{3 / 2}+O\left(P_{\mathrm{err}} t^{2}\right),
$$

where

$$
\begin{aligned}
P & =\frac{1}{|\Omega|} \int_{\Gamma}\left(\frac{4}{3 \sqrt{\pi}} \sqrt{\mathcal{D}_{0}}\left(\mathbf{u}_{\mathbf{g}} \cdot \nu(\mathbf{x})\right)^{2}\right) d s_{\mathbf{x}} \\
P_{\text {err }} & =-\frac{\mathcal{D}_{0}}{4|\Omega|} \int_{\Gamma} k(\mathbf{x})\left(\mathbf{u}_{\mathbf{g}} \cdot \nu(\mathbf{x})\right)^{2} d s_{\mathbf{x}}
\end{aligned}
$$

and

$$
I=\frac{\mathcal{D}_{0}}{\delta^{2}\left(\Delta-\frac{\delta}{3}\right)} \int_{0}^{\delta} t h(t) d t=\frac{2 \mathcal{D}_{0} P}{7\left(\Delta-\frac{\delta}{3}\right)} \delta^{7 / 2}+O\left(\mathcal{D}_{0} P_{\text {err }} \frac{\delta^{2}}{4\left(\Delta-\frac{\delta}{3}\right)}\right) .
$$

Between the pulses, we obtain

(69)

$$
h(t)=\frac{1}{|\Omega|} \int_{\Gamma} \omega(\mathbf{x}, t)\left(\mathbf{u}_{\mathbf{g}} \cdot \nu(\mathbf{x})\right) d s_{\mathbf{x}}=P\left(t^{3 / 2}-(t-\delta)^{3 / 2}\right)+O\left(P_{\mathrm{err}}\left(t^{2}-(t-\delta)^{2}\right)\right)
$$

and

$$
\begin{aligned}
I I= & \frac{\mathcal{D}_{0}}{\delta^{2}\left(\Delta-\frac{\delta}{3}\right)} \int_{\delta}^{\Delta} \delta h(t) d t=-\frac{2}{5} \frac{\mathcal{D}_{0} P\left(\delta^{7 / 2}-\Delta^{5 / 2} \delta+(\Delta-\delta)^{5 / 2} \delta\right)}{\delta^{2}\left(\Delta-\frac{\delta}{3}\right)} \\
& +O\left(\mathcal{D}_{0} P_{\text {err }}\left(\frac{\Delta^{2}-\delta \Delta}{\Delta-\frac{\delta}{3}}\right)\right) .
\end{aligned}
$$


During the second pulse, we find

$$
\begin{aligned}
h(t)= & \frac{1}{|\Omega|} \int_{\Gamma} \omega(\mathbf{x}, t)\left(\mathbf{u}_{\mathbf{g}} \cdot \nu(\mathbf{x})\right) d s_{\mathbf{x}}=P\left(t^{3 / 2}-(t-\delta)^{3 / 2}-(t-\Delta)^{3 / 2}\right) \\
& +O\left(P_{\text {err }}\left(t^{2}-(t-\delta)^{2}-(t-\Delta)^{2}\right)\right)
\end{aligned}
$$

and

$$
\begin{aligned}
I I I= & \frac{\mathcal{D}_{0}}{\delta^{2}\left(\Delta-\frac{\delta}{3}\right)} \int_{\Delta}^{\Delta+\delta}(\Delta+\delta-t) h(t) d t \\
= & \frac{2}{35} \frac{\mathcal{D}_{0} P}{\delta^{2}\left(\Delta-\frac{\delta}{3}\right)}\left(\left(2 \Delta^{3}+\Delta^{2} \delta-8 \Delta \delta^{2}+5 \delta^{3}\right) \sqrt{\Delta-\delta}+2(\Delta+\delta)^{7 / 2}\right. \\
& \left.\quad-4 \Delta^{7 / 2}-7 \Delta^{5 / 2} \delta-2 \delta^{7 / 2}\right)+O\left(\mathcal{D}_{0} P_{\text {err }}\left(\frac{\Delta \delta-\frac{1}{4} \delta^{2}}{\Delta-\frac{\delta}{3}}\right)\right) .
\end{aligned}
$$

Finally, adding up the above expressions, we obtain that using the layer potentials representation,

$$
\begin{aligned}
D_{\mathbf{u}_{\mathrm{g}}}^{\mathrm{eff}}=\mathcal{D}_{0} & {\left[1-\frac{4}{35} \frac{P}{\delta^{2}\left(\Delta-\frac{\delta}{3}\right)}\left((\Delta+\delta)^{7 / 2}-2\left(\delta^{7 / 2}+\Delta^{7 / 2}\right)+(\Delta-\delta)^{7 / 2}\right)\right] } \\
& +O\left(\mathcal{D}_{0} P_{\mathrm{err}} \frac{\Delta^{2}}{\Delta-\frac{\delta}{3}}\right)
\end{aligned}
$$

with $P$ and $P_{\text {err }}$ defined in (66) and (67), respectively.

We observe that, in the narrow pulse limit, $\delta \ll \Delta$, the expression (73)

$$
D_{\mathbf{u}_{\mathrm{g}}}^{\mathrm{eff}}=\mathcal{D}_{0}\left(1-\frac{4}{3 \sqrt{\pi}} \sqrt{\mathcal{D}_{0} \Delta} \frac{\int_{\Gamma}\left(\mathbf{u}_{\mathrm{g}} \cdot \nu\right)^{2} d s_{\mathbf{x}}}{|\Omega|}\right)+O\left(\mathcal{D}_{0} P_{\mathrm{err}} \Delta\right),
$$

reduces to the formula given in $[1,9]$. If $\Omega$ is an isotropic domain, then

$$
\frac{\int_{\Gamma}\left(\mathbf{u}_{\mathbf{g}} \cdot \nu\right)^{2} d s_{\mathbf{x}}}{|\Omega|}=\frac{|\Gamma|}{d|\Omega|},
$$

which is the ratio of surface to volume divided by the space dimension $d$, exactly the quantity contained in the formula in [27]. Hence our new formula in (73) is a correction of the results in $[1,9,27]$ because it takes into account the contribution of $\delta$. This makes the new formula applicable for cases where the narrow pulse assumption $\delta \ll \Delta$ does not hold. Of course, this description still hold only for short times due to the nature of the asympotic expansions for layer potentials.

3.3. Mixed approximation. When the pulses are short but the delay between the pulses is not short (with respect to diffusion in $\Omega$ ), we use the single layer potential representation in the first and third intervals and the eigenfunction representation between the pulses.

In the first pulse, $t \in[0, \delta]$, we have the same results as in the previous section, namely,

$$
I=\frac{8}{21 A|\Omega| \sqrt{\pi}} \mathcal{D}_{0}^{3 / 2} \delta^{7 / 2} \int_{\Gamma}\left(\mathbf{u}_{\mathbf{g}} \cdot \nu\right)^{2} d s_{\mathbf{x}}+O\left(\frac{\delta^{2}}{\left(\Delta-\frac{\delta}{3}\right)}\right) .
$$


Between the pulses, $t \in[\delta, \Delta]$, the Neumann boundary condition in (18) is

$$
\mathcal{D}_{0} \nabla \omega(\mathbf{x}, t) \cdot \nu=\mathcal{D}_{0} \delta \mathbf{u}_{\mathrm{g}} \cdot \nu, \quad \text { on } \Gamma \times[\delta, \Delta]
$$

and the initial condition is

$$
\omega(\mathbf{x}, \delta)=S\left[2 \delta \mathbf{u}_{\mathbf{g}} \cdot \nu\right](\mathbf{x}, t)+O\left(\delta^{2}\right), \quad \mathbf{x} \text { in } \Omega .
$$

The function $\widetilde{\omega}(\mathbf{x}, t)=\omega(\mathbf{x}, t)-\delta \mathbf{x} \cdot \mathbf{u}_{\mathbf{g}}$ satisfies homogeneous Neumann boundary condition and the initial condition

$$
\widetilde{\omega}(\mathbf{x}, \delta)=S\left[\left(2 \delta \mathbf{u}_{\mathbf{g}} \cdot \nu\right)\right](\mathbf{x}, \delta)-\delta \mathbf{x} \cdot \mathbf{u}_{\mathbf{g}}
$$

This means

$$
\widetilde{\omega}(\mathbf{x}, t)=c_{0}+\sum_{n=1}^{\infty} c_{n} e^{-\lambda_{n} \mathcal{D}_{0}(t-\delta)} \phi_{n}(\mathbf{x})
$$

where

$$
\begin{aligned}
& \left.c_{0}=-\delta a_{0}+b_{0}=-\frac{\delta}{|\Omega|} \int_{\Omega} \mathbf{x} \cdot \mathbf{u}_{\mathbf{g}} d \mathbf{x}+\frac{1}{|\Omega|} \int_{\Omega} \omega(\mathbf{x}, \delta) d \mathbf{x}\right), \\
& c_{n}=-\delta a_{n}+b_{n}=-\delta \int_{\Omega} \mathbf{x} \cdot \mathbf{u}_{\mathbf{g}} \phi_{n}(\mathbf{x}) d \mathbf{x}+\int_{\Omega} \omega(\mathbf{x}, \delta) \phi_{n}(\mathbf{x}) d \mathbf{x}
\end{aligned}
$$

with again $\phi_{n}$ and $\lambda_{n}$ the Neumann eigenfunctions and eigenvalues associated to the Laplace operator $(n=1,2, \ldots)$. Thus, for $t \in[\delta, \Delta]$,

$$
\omega(\mathbf{x}, t)=c_{0}+\sum_{n=1}^{\infty} c_{n} e^{-\lambda_{n} \mathcal{D}_{0}(t-\delta)} \phi_{n}(\mathbf{x})+\delta \mathbf{x} \cdot \mathbf{u}_{\mathbf{g}}+O\left(\delta^{2}\right),
$$

and

$$
h(t)=\sum_{n=1}^{\infty} \frac{c_{n} \lambda_{n} a_{n}}{|\Omega|} e^{-\lambda_{n} \mathcal{D}_{0}(t-\delta)}+\delta+O\left(\frac{\delta^{2}}{A}\right),
$$

and

$$
I I=\frac{1}{A} \sum_{n=1}^{\infty} \frac{c_{n} a_{n}}{|\Omega|}\left(1-e^{-\lambda_{n} \mathcal{D}_{0}(\Delta-\delta)}\right)+\frac{\mathcal{D}_{0} \delta^{2}(\Delta-\delta)}{A}+O\left(\frac{\delta(\Delta-\delta)}{\left(\Delta-\frac{\delta}{3}\right)}\right) .
$$

During the second pulse, $t \in[\Delta, \Delta+\delta]$, we keep the solution from the previous interval in Eq. (83) which satisfies homogeneous boundary conditions and just add a single layer potential to match the Neumann boundary condition. We obtain

$$
\begin{aligned}
\omega(\mathbf{x}, t)=c_{0}+\sum_{n=1}^{\infty} c_{n} e^{-\lambda_{n} \mathcal{D}_{0}(t-\delta)} \phi_{n}(\mathbf{x}) & +\delta \mathbf{x} \cdot \mathbf{u}_{\mathbf{g}} \\
& +S\left[\left(-2 \tau \mathbf{u}_{\mathbf{g}} \cdot \nu\right)\right](\mathbf{x}, t-\Delta)+O\left((t-\Delta)^{2}\right),
\end{aligned}
$$

where $t \in[\Delta, \Delta+\delta]$. The density in the single layer potential is $-2 \tau \mathbf{u}_{\mathbf{g}} \cdot \nu$ with $\tau \in[0, \delta]$ from a shift in time $\tau=t-\Delta$. Similar reasoning as in the previous sections gives

$$
S\left[\left(-2 \tau \mathbf{u}_{\mathbf{g}} \cdot \nu\right)\right](\mathbf{x}, t-\Delta)=-\frac{4}{3 \sqrt{\pi}|\Omega|} \sqrt{\mathcal{D}_{0}}(t-\Delta)^{3 / 2} \int_{\Gamma}\left(\mathbf{u}_{\mathbf{g}} \cdot \nu\right)+O\left((t-\Delta)^{5 / 2}\right),
$$


which leads to

$$
\begin{array}{r}
h(t)=\sum_{n=1}^{\infty} \frac{c_{n}}{|\Omega|} e^{-\lambda_{n} \mathcal{D}_{0}(t-\delta)} \lambda_{n} a_{n}-\frac{4}{3 \sqrt{\pi}|\Omega|} \sqrt{\mathcal{D}_{0}}(t-\Delta)^{3 / 2} \int_{\Gamma}\left(\mathbf{u}_{\mathbf{g}} \cdot \nu\right)^{2} d s_{\mathbf{x}} \\
+\delta+O\left(\frac{(t-\Delta)^{2}}{A}\right),
\end{array}
$$

and

$$
\begin{aligned}
I I I=\frac{\mathcal{D}_{0}}{2 A} \delta^{3}+ & \sum_{n=1}^{\infty} \frac{c_{n} a_{n}}{\mathcal{D}_{0} \lambda_{n} A|\Omega|}\left(e^{-\lambda_{n} \mathcal{D}_{0}(\Delta-\delta)}\left(\mathcal{D}_{0} \lambda_{n} \delta-1\right)+e^{-\lambda_{n} \mathcal{D}_{0} \Delta}\right) \\
& -\frac{16}{105 \sqrt{\pi} A|\Omega|} \mathcal{D}_{0}^{3 / 2}\left(\int_{\Gamma}\left(\mathbf{u}_{\mathbf{g}} \cdot \nu\right)^{2} d s_{\mathbf{x}}\right) \delta^{7 / 2}+O\left(\frac{\delta^{2}}{\left(\Delta-\frac{\delta}{3}\right)}\right) .
\end{aligned}
$$

The effective diffusion coefficient for the compartment $\Omega$ assumes thus the form

$$
\begin{aligned}
D_{\mathbf{u}_{\mathbf{g}}}^{\mathrm{eff}}=\frac{\mathcal{D}_{0} \delta}{6\left(\Delta-\frac{\delta}{3}\right)}-\frac{8 \mathcal{D}_{0}^{3 / 2} \delta^{3 / 2}}{35 \sqrt{\pi}|\Omega|\left(\Delta-\frac{\delta}{3}\right)} \int_{\Gamma}\left(\mathbf{u}_{\mathbf{g}} \cdot \nu\right)^{2} d s_{\mathbf{x}}+ & \\
-\sum_{n=1}^{\infty} \frac{-\delta\left(a_{n}\right)^{2}+a_{n} b_{n}}{|\Omega| \delta^{2}\left(\Delta-\frac{\delta}{3}\right)}\left(\delta-\frac{e^{-\lambda_{n} \mathcal{D}_{0} \Delta}\left(1-e^{\lambda_{n} \mathcal{D}_{0} \delta}\right)}{\lambda_{n} \mathcal{D}_{0}}\right) & \left(\max \left\{\frac{\delta^{2}}{\left(\Delta-\frac{\delta}{3}\right)}, \frac{\delta(\Delta-\delta)}{\left(\Delta-\frac{\delta}{3}\right)}\right\}\right) \\
& \quad+O
\end{aligned}
$$

In the narrow pulse limit, we get

$$
D_{\mathbf{u}_{\mathrm{g}}}^{\mathrm{eff}} \approx \sum_{n=1}^{\infty} \frac{\left(\delta a_{n}-b_{n}\right) a_{n}}{|\Omega| \delta\left(\Delta-\frac{\delta}{3}\right)}\left(1-e^{-\lambda_{n} \mathcal{D}_{0} \Delta}\right),
$$

which again tells us that $D_{\mathbf{u}_{\mathrm{g}}}^{\text {eff }}$ approaches its long time limit as $1 / \Delta$, because $b_{n}=$ $O\left(\delta^{3 / 2}\right)$ for all $n \geq 1$ due to the maximum principle for heat equation applied to $\omega(\mathbf{x}, t)$ in the first pulse:

$$
\|\omega(\mathbf{x}, t)\| \leq\left\|\omega\left(\mathbf{x}^{0}, t\right)\right\| \approx O\left(t^{3 / 2}\right) \quad \forall \mathbf{x} \in \Omega, \mathbf{x}^{0} \in \Gamma, t \in[0, \delta] .
$$

4. Averaging $D^{\text {eff }}$ over multiple gradient directions. If we average the effective diffusion coefficient $D_{\mathbf{u}_{\mathrm{g}}}^{\text {eff }}$ over all the possible gradient directions $\mathbf{u}_{\mathbf{g}}$, we can obtain a new formula that is independent of the orientation of the biological cells. We define the orientionally averaged effective diffusion coefficient as

$$
D_{\text {ave }}^{\mathrm{eff}}:=\frac{\int_{\mathbb{S}^{d-1}} D_{\mathbf{u}_{\mathbf{g}}}^{\mathrm{eff}} d \mathbf{u}}{\int_{\mathbb{S}^{d-1}} d \mathbf{u}} .
$$

We recall that

$$
D_{\mathbf{u}_{\mathbf{g}}}^{\mathrm{eff}}=\mathcal{D}_{0}-\frac{\mathcal{D}_{0}}{A|\Omega|} \int_{0}^{T E} F(t) \int_{\Omega} \mathbf{u}_{\mathbf{g}} \cdot \nabla \omega_{\mathbf{u}_{\mathbf{g}}}(\mathbf{x}, t) d \mathbf{x} d t
$$


where $\omega_{\mathbf{u}_{\mathbf{g}}}(\mathbf{x}, t)$ solves the problem (17). Because of the linearity of the Neumann problem, for every direction $\mathbf{u}_{\mathbf{g}}=\left[u_{1}, \ldots, u_{d}\right]$ we have that

$$
\omega_{\mathbf{u}_{\mathbf{g}}}(\mathbf{x}, t)=\sum_{i=1}^{d} u_{i} \omega_{\mathbf{e}_{i}}(\mathbf{x}, t),
$$

where $\mathbf{e}_{i}$ is the $i$-th vector of the canonical basis of $\mathbb{R}^{d}$. As a consequence

$$
\begin{aligned}
D_{\mathbf{u g}}^{\mathrm{eff}} & =\mathcal{D}_{0}-\frac{\mathcal{D}_{0}}{A|\Omega|} \int_{0}^{T E} F \int_{\Omega}\left(u_{1} \mathbf{e}_{1}+\cdots+u_{d} \mathbf{e}_{d}\right) \cdot\left(u_{1} \nabla \omega_{\mathbf{e}_{1}}+\cdots+u_{d} \nabla \omega_{\mathbf{e}_{i}}\right) d \mathbf{x} d t \\
& =\mathcal{D}_{0}-\frac{\mathcal{D}_{0}}{A|\Omega|} \int_{0}^{T E} F\left(\sum_{i=1}^{d} u_{i}^{2} \int_{\Omega} \mathbf{e}_{i} \cdot \nabla \omega_{\mathbf{e}_{i}} d \mathbf{x}+\sum_{\substack{i \neq j \\
i, j=1}}^{d} u_{i} u_{j} \int_{\Omega} \mathbf{e}_{i} \cdot \nabla \omega_{\mathbf{e}_{j}} d \mathbf{x}\right) d t
\end{aligned}
$$

and thus, if we want to average over all the possible directions, we are interested in the integrals

$$
\frac{\int_{\mathbb{S}^{d-1}} u_{i}^{2} d \mathbf{u}}{\int_{\mathbb{S}^{d-1}} d \mathbf{u}}, i=1, \ldots, d \quad \text { and } \quad \frac{\int_{\mathbb{S}^{d-1}} u_{i} u_{j} d \mathbf{u}}{\int_{\mathbb{S}^{d-1}} d \mathbf{u}}, i, j=1, \ldots, d, i \neq j .
$$

We observe that, for all $i, j=1, \ldots, d$ and $i \neq j$,

$$
\int_{\mathbb{S}^{d-1}} u_{i} u_{j} d \mathbf{u}=0
$$

Therefore, what remains in the average are just the terms

$$
\sum_{i=1}^{d} \frac{\int_{\mathbb{S}^{d-1}} u_{i}^{2} d \mathbf{u}}{\int_{\mathbb{S}^{d-1}} d \mathbf{u}} \int_{\Omega} \mathbf{e}_{i} \cdot \nabla \omega_{\mathbf{e}_{i}} d \mathbf{x}
$$

i.e. simply the average over $d$ perpendicular directions and then

$$
D_{\text {ave }}^{\mathrm{eff}}=\mathcal{D}_{0}-\sum_{i=1}^{d} \frac{\mathcal{D}_{0}}{d A|\Omega|} \int_{0}^{T E} F(t) \int_{\Omega} \mathbf{u}_{\mathbf{g}}{ }^{i} \cdot \nabla \omega_{\mathbf{u}_{\mathbf{g}} i}(\mathbf{x}, t) d \mathbf{x} d t
$$

where $\mathbf{u}_{\mathbf{g}}{ }^{i}, i=1, \ldots, d$ are $d$ orthogonal directions. In short, averaging over all the possible directions is equivalent to average only over $d$ orthogonal normalized directions.

We use the fact that

$$
\sum_{i=i}^{d} \frac{\int_{\Gamma}\left(\mathbf{u}_{\mathbf{g}}{ }^{i} \cdot \nu\right)^{2} d s_{\mathbf{x}}}{d}=\frac{|\Gamma|}{d}
$$

and we define

$$
k_{n}:=\sum_{i=1}^{d} \frac{-\left(a_{n}^{i}\right)^{2}}{d|\Omega|}=\sum_{i=1}^{d} \frac{-\left(\int_{\Omega} \mathbf{x} \cdot \mathbf{u}_{\mathbf{g}}{ }^{i} \phi_{n}(\mathbf{x}) d \mathbf{x}\right)^{2} d \mathbf{u}_{\mathbf{g}}}{d|\Omega|},
$$

i.e. the mean over $d$ orthogonal directions of the square of the first moment along these directions, and

$$
j_{n}:=\sum_{i=1}^{d} \frac{b_{n}^{i} a_{n}^{i}}{d|\Omega|}=\sum_{i=1}^{d} \frac{\left(\int_{\Omega} \omega_{\mathbf{u}_{\mathbf{g}}{ }^{i}}(\mathbf{x}, \delta) \phi_{n}(\mathbf{x}) d \mathbf{x}\right)\left(\int_{\Omega} \mathbf{x} \cdot \mathbf{u}_{\mathbf{g}}{ }^{i} \phi_{n}(\mathbf{x}) d \mathbf{x}\right)}{d|\Omega|} .
$$


In summary, the eigenfunction expansion in (36) gives

$$
\begin{aligned}
D_{\text {ave }}^{\mathrm{eff}}=\sum_{n=1}^{\infty} \frac{k_{n}}{\mathcal{D}_{0}^{2} \lambda_{n}^{2} \delta^{2}\left(\Delta-\frac{\delta}{3}\right)}\left[e^{-\mathcal{D}_{0} \lambda_{n}(\Delta+\delta)}+e^{-\mathcal{D}_{0} \lambda_{n}(\Delta-\delta)}\right. & \\
& \left.-2\left(\mathcal{D}_{0} \lambda_{n} \delta+e^{-\mathcal{D}_{0} \lambda_{n} \delta}+e^{-\mathcal{D}_{0} \lambda_{n} \Delta}-1\right)\right]
\end{aligned}
$$

The single layer potential representation gives

$$
D_{\text {ave }}^{\text {eff }}
$$

$$
\begin{aligned}
(103)= & \mathcal{D}_{0}-\frac{16}{35} \frac{\mathcal{D}_{0}^{3 / 2}}{\delta^{2}(3 \Delta-\delta) \sqrt{\pi}}\left[(\Delta-\delta)^{7 / 2}+(\Delta+\delta)^{7 / 2}-2\left(\delta^{7 / 2}+\Delta^{7 / 2}\right)\right] \frac{|\Gamma|}{d|\Omega|} \\
& +O(\Delta) .
\end{aligned}
$$

The mixed approximation in (89) gives

$$
\begin{aligned}
D_{\text {ave }}^{\mathrm{eff}}=\frac{\mathcal{D}_{0} \delta}{6\left(\Delta-\frac{\delta}{3}\right)}- & \frac{8 \mathcal{D}_{0}^{3 / 2} \delta^{3 / 2}}{35 \sqrt{\pi}\left(\Delta-\frac{\delta}{3}\right)} \frac{|\Gamma|}{d|\Omega|} \\
-\sum_{n=1}^{\infty} \frac{\delta k_{n}+j_{n}}{\delta^{2}\left(\Delta-\frac{\delta}{3}\right)}\left(\delta-\frac{e^{-\lambda_{n} \mathcal{D}_{0} \Delta}\left(1-e^{\lambda_{n} \mathcal{D}_{0} \delta}\right)}{\lambda_{n} \mathcal{D}_{0}}\right) & \left(\max \left\{\frac{\delta^{2}}{\left(\Delta-\frac{\delta}{3}\right)}, \frac{\delta(\Delta-\delta)}{\left(\Delta-\frac{\delta}{3}\right)}\right\}\right) .
\end{aligned}
$$

5. Numerical results. In this Section we numerically validate the approximate formulas we derived in the previous sections. To compute the reference quantities we solved the diffusion equation in (17) using the Matlab PDEToolbox. The eigenvalues and eigenfunctions of the Laplace equation with Neumann boundary conditions were also computed with the same software. The convergence between the $\mathrm{H}-A D C$ model and the Bloch-Torrey equation was shown previously in [14].

First we show the three approximations of $h(t)$. We consider a 2D geometry of one vertically orientated ellipse with semi-axes of $19 \mu \mathrm{m}$ and $9 \mu \mathrm{m}$. The intrinsic diffusion coefficient is set to $\mathcal{D}_{0}=1 e^{-3} \mathrm{~mm}^{2} / \mathrm{s}$ and we vary the values of $\delta, \Delta$ and $\mathbf{u}_{\mathrm{g}}$. To compute the reference solution $h(t)$ we solved the problem (17) on the finite element mesh shown in Fig. 2. The eigenvalues and eigenfunctions are also computed on the same finite element mesh. The projections $a_{n}$ and $b_{n}$ are computed according the the formulas in (25) and (81).

For this particular geometry the first four non-zero eigenvalues are

$$
\lambda_{1}=0.0097, \quad \lambda_{2}=0.0325, \quad \lambda_{3}=0.0383, \quad \lambda_{4}=0.0644,
$$

and their numerically calculated projections $a_{i}$ are reported in the table below.

Clearly, among the four eigenvalues, in the direction $\mathbf{u}_{\mathbf{g}}=[1,0]$, all but $\lambda_{3}$ have negligible contribution, and in the direction $\mathbf{u}_{\mathrm{g}}=[0,1]$, all but $\lambda_{1}$ have negligible contribution.

In the following plots we always indicate the reference quantity with a line, the single layer approximation with squares, the eigenfunction approximation with circles and the mixed approximation with asterisks.

In Figure 3 we considered $\delta=5 \mathrm{~ms}, \Delta=10 \mathrm{~ms}$ and $\mathbf{u}_{\mathbf{g}}=[1,0]$. As we can see the single layer approximation (squares) fits very well the reference quantity (continuous line) in all three time intervals. We also notice that the mixed approximation 


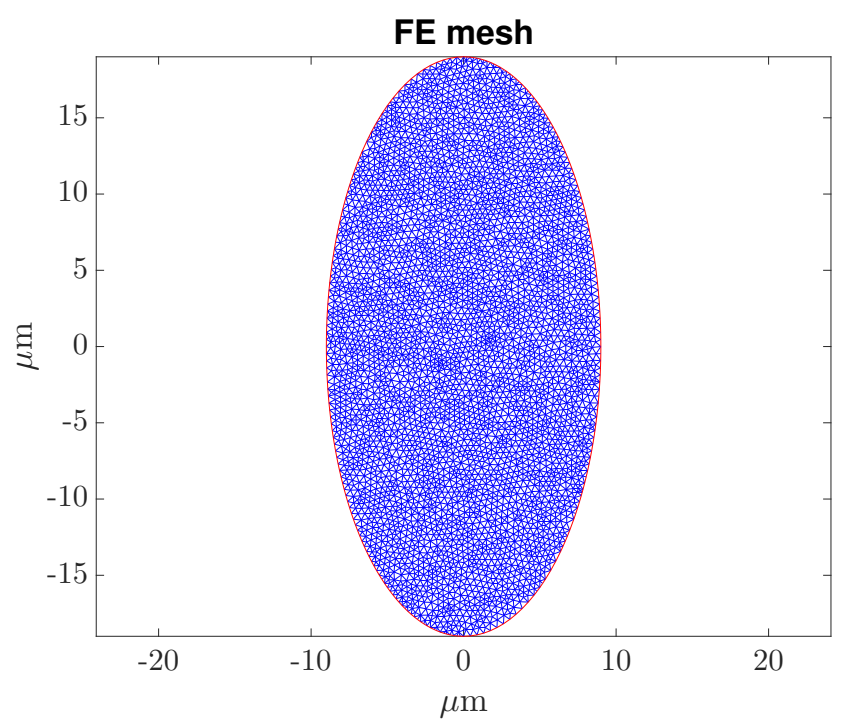

Fig. 2: Finite elements mesh of an ellipse with semiaxes of $19 \mu \mathrm{m}$ and $9 \mu \mathrm{m}$, orientated vertically along the $y$-axis.

\begin{tabular}{ccccc}
$\mathbf{u}_{\mathbf{g}}$ & $a_{1}$ & $a_{2}$ & $a_{3}$ & $a_{4}$ \\
\hline$[1,0]$ & 38.9 & -25.7 & $-4.62 e^{+5}$ & -0.97 \\
{$[0,1]$} & $1.07 e^{+6}$ & 1.75 & -4.49 & -5.58
\end{tabular}

Table 1: The first moments of the eigenfunction associated with the first four non-zero eigenvalues in the two directions $\mathbf{u}_{\mathbf{g}}=[1,0]$ and $\mathbf{u}_{\mathbf{g}}=[0,1]$.

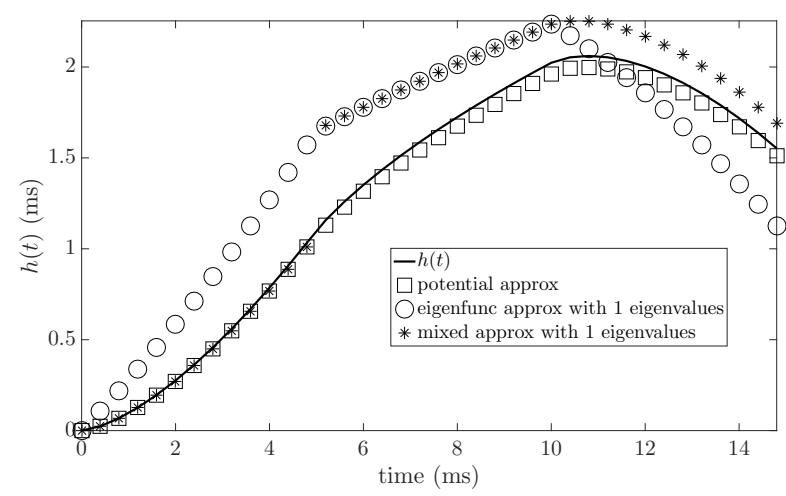

Fig. 3: $h(t)$ and its approximation (using the three different formulas found) with respect to the gradient directions $\mathbf{u}_{\mathbf{g}}=[1,0]$ for an ellipse of semi-axes $19 \mu \mathrm{m}$ and $9 \mu \mathrm{m}$. Intrinsic diffusion coefficients $\mathcal{D}_{0}=1 \times 10^{-3} \mathrm{~mm}^{2} / \mathrm{s}$, pulses duration $\delta=5 \mathrm{~ms}$ and time-delay between pulses $\Delta=10 \mathrm{~ms}$. 


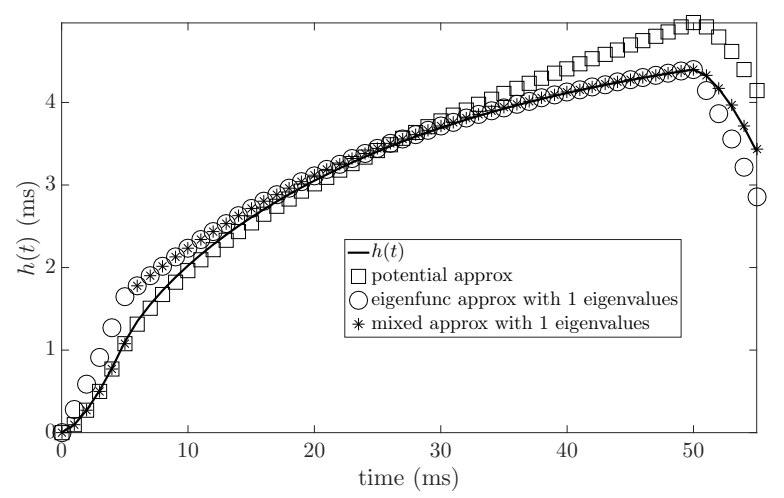

Fig. 4: $h(t)$ and its approximation (using the three different formulas found) with respect to the gradient directions $\mathbf{u}_{\mathrm{g}}=[1,0]$ for an ellipse of semiaxes $19 \mu \mathrm{m}$ and $9 \mu \mathrm{m}$. Intrinsic diffusion coefficients $\mathcal{D}_{0}=1 \times 10^{-3} \mathrm{~mm}^{2} / \mathrm{s}$, pulses duration $\delta=5 \mathrm{~ms}$ and time-delay between pulses $\Delta=50 \mathrm{~ms}$.

(asterisks) works sufficiently well during the two pulses but not between them. For the eigenfunctions approximation, the fit is far from the reference quantity.

In Figure 4 we considered $\delta=5 \mathrm{~ms}, \Delta=50 \mathrm{~ms}$ and $\mathbf{u}_{\mathbf{g}}=[1,0]$. As we can see the single layer approximation fits well the reference quantity during the first pulse and until $t \approx 25 \mathrm{~ms}$, but after that, the approximation is no longer good. The eigenfunction approximation is not good during the pulses but it becomes accurate at the end of the interval between them. The mixed approximation fits well during the pulses and is the same as the eigenfunction approximation between the pulses.

In Figures $5 \mathrm{a}$ and $5 \mathrm{~b}$ we show the behaviour of $D^{\mathrm{eff}}$ computed for two different directions of the gradient $\left(\mathbf{u}_{\mathrm{g}}=[1,0]\right.$ and $\left.\mathbf{u}_{\mathrm{g}}=[0,1]\right)$ but the same parameters ( $\delta=5 \mathrm{~ms}$ and thirty different values of $\Delta$ equally distributed in the interval $[8,80] \mathrm{ms}$ ). In Figure $5 \mathrm{c}$ we show the average of $D_{\text {ave }}^{\text {eff }} / \mathcal{D}_{0}$ along the two perpendicular directions. Clearly, the single layer formula works well for at short $\Delta+\delta$, the eigenfunctions formula for long $\Delta+\delta$.

To conclude, in Figure 6 we report the absolute error

$$
\left|D^{\mathrm{eff}}-D^{\text {approx }}\right|
$$

for the same parameters as before in the two orthogonal directions. As we can see, the single layer approximation is better at short times and the eigenfunctions approximation is better at long times. The time at which the switch between the short and the long diffusion time approximations occurs at around $t=50 \mathrm{~ms}$ in the direction $[1,0]$ and it occurs at around $t=25 \mathrm{~ms}$ in the direction $[0,1]$.

6. Conclusions. Diffusion Magnetic Resonance Imaging (dMRI) can be used to measure a time and direction dependent effective diffusion coefficient which can in turn reveal information about the tissue micro-structure. Recently a new mathematical model for the effective diffusion coefficient, the $\mathrm{H}-A D C$ model, was obtained using homogenization techniques after imposing a certain scaling relationship between the physical parameters. The resulting model depends on the solution of a diffusion equation subject to time-dependent Neumann boundary conditions. 


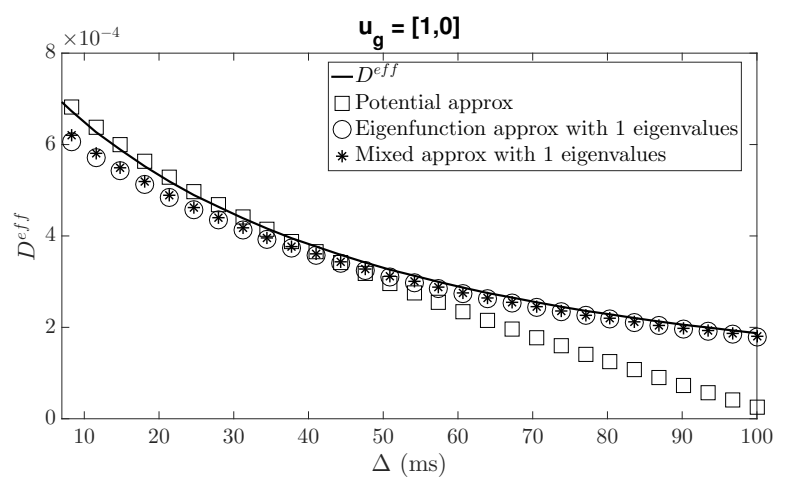

(a) Gradient direction $\mathbf{u}_{\mathbf{g}}=[1,0]$

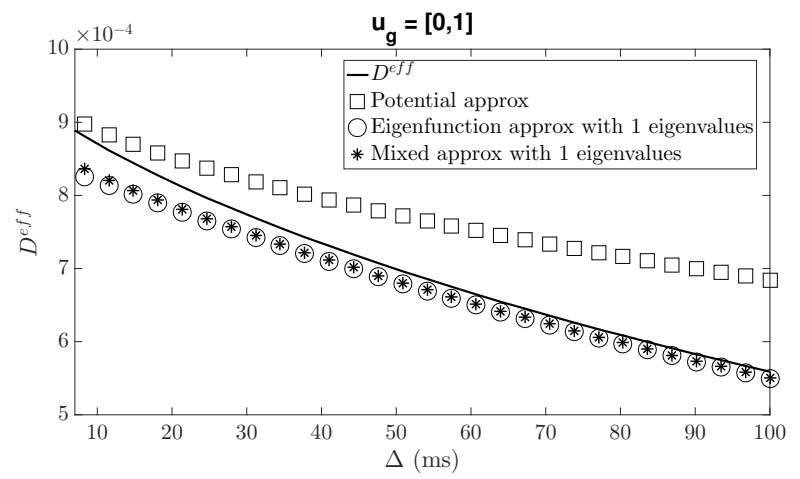

(b) Gradient direction $\mathbf{u}_{\mathrm{g}}=[0,1]$

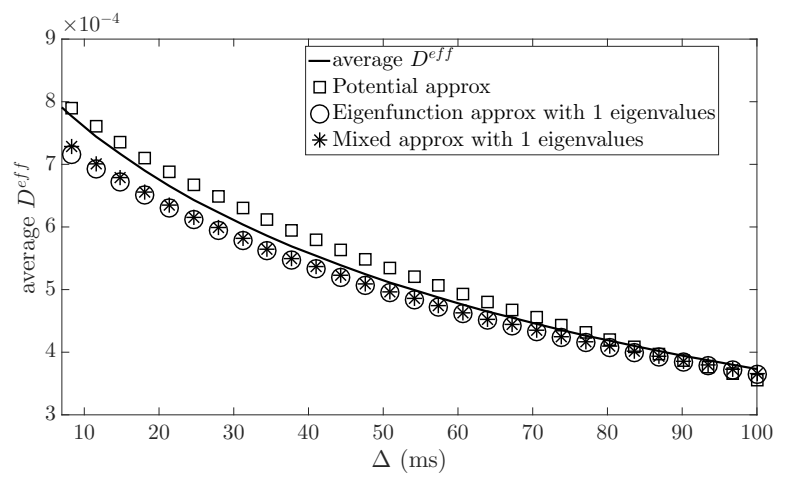

(c) Average over both directions

Fig. 5: $D^{\text {eff }}$ with respect to two different gradient directions as well as $D_{\text {ave }}^{\text {eff }}$, the average over both direction, compared to approximations using the three different formulas, for an ellipse of semiaxes $19 \mu \mathrm{m}$ and $9 \mu \mathrm{m}$. Intrinsic diffusion coefficients $\mathcal{D}_{0}=1 \times 10^{-3} \mathrm{~mm}^{2} / \mathrm{s}$, pulses duration $\delta=5 \mathrm{~ms}$ and thirty different values of the time-delay between pulses in the interval $[8,80] \mathrm{ms}$.

In this paper, we analysed the $\mathrm{H}-A D C$ model in the case of finite sub-domains.

In particular, we obtained three representations of the effective diffusion coefficient 


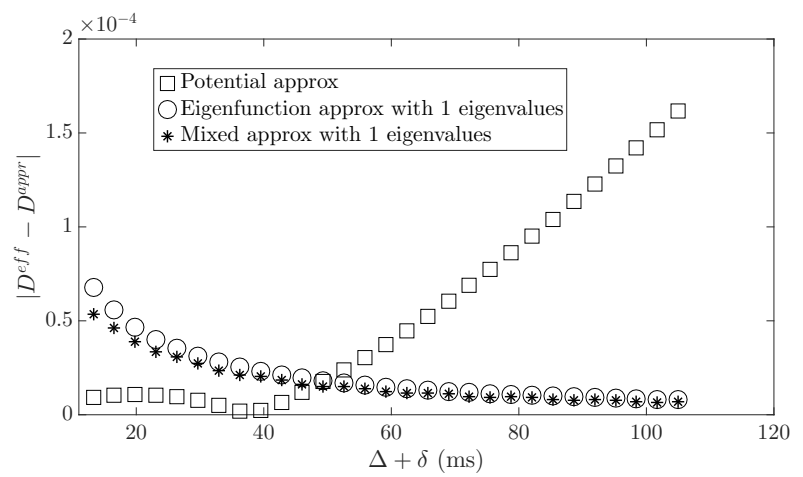

(a) Gradient direction $\mathbf{u}_{\mathrm{g}}=[1,0]$

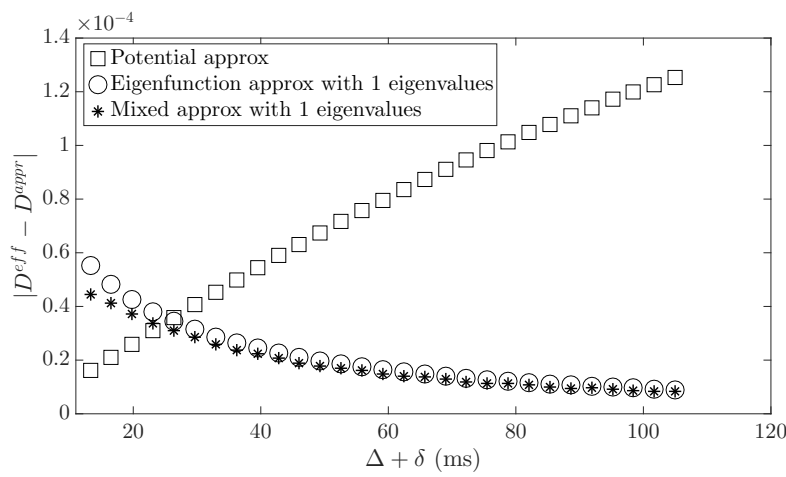

(b) Gradient direction $\mathbf{u}_{\mathbf{g}}=[0,1]$

Fig. 6: Absolute error $\left|D^{\text {eff }}-D^{\text {approx }}\right|$ using the three different formulas found with respect to two different gradient directions for an ellipse of semi-axes $19 \mu \mathrm{m}$ and $9 \mu \mathrm{m}$. Intrinsic diffusion coefficients $\mathcal{D}_{0}=1 \times 10^{-3} \mathrm{~mm}^{2} / \mathrm{s}$, pulses duration $\delta=5 \mathrm{~ms}$ and thirty different values of the time-delay between pulses in the interval $[8,80] \mathrm{ms}$.

that are appropriate in different diffusion time regimes. In the short time regime, we proposed using a representation based on the single layer potential. In the long time regime when the pulse duration is not short, we proposed using a representation based on the eigenfunctions expansion of the Neumann Laplace operator. In the long time regime when the pulse duration is short, we proposed a representation that combines the single layer potential during the pulses with the eigenfunction expansion between the pulses. In particular, in the short time regime, our representation corrects an existing formula by correctly accounting for the pulse duration. Our work helps to make more precise how parameters of the tissue micro-structure such as the surface to volume ratio or the dominant eigenvalue and its projection affect the effective diffusion coefficient.

\section{REFERENCES}

[1] S. Axelrod And P. N. Sen, Nuclear magnetic resonance spin echoes for restricted diffusion in an inhomogeneous field: Methods and asymptotic regimes, The Journal of Chemical Physics, 114 (2001), p. 6878, http://link.aip.org/link/?JCPSA6/114/6878/1. 
[2] A. V. BARZYKIN, Theory of spin echo in restricted geometries under a step-wise gradient pulse sequence, Journal of Magnetic Resonance, 139 (1999), pp. 342-353, http://www. sciencedirect.com/science/article/pii/S1090780799917780.

[3] L. M. Burcaw, E. Fieremans, AND D. S. Novikov, Mesoscopic structure of neuronal tracts from time-dependent diffusion, NeuroImage, 114 (2015), pp. 18 - 37, https: //doi.org/http://dx.doi.org/10.1016/j.neuroimage.2015.03.061, http://www.sciencedirect. com/science/article/pii/S1053811915002578.

[4] P. T. Callaghan, A. Coy, D. MacGowan, K. J. Packer, and F. O. Zelaya, Diffraction-like effects in nmr diffusion studies of fluids in porous solids, Nature, 351 (1991), pp. 467-469, http://dx.doi.org/10.1038/351467a0.

[5] J. Chen, W. Liu, H. Zhang, L. Lacy, X. Yang, S.-K. Song, S. A. Wickline, and $\mathrm{X}$. YU, Regional ventricular wall thickening reflects changes in cardiac fiber and sheet structure during contraction: quantification with diffusion tensor MRI, American Journal of Physiology - Heart and Circulatory Physiology, 289 (2005), pp. H1898-H1907, https://doi.org/10.1152/ajpheart.00041.2005.

[6] J. Chen, S.-K. Song, W. Liu, M. Mclean, J. S. Allen, J. Tan, S. A. Wickline, and X. Yu, Remodeling of cardiac fiber structure after infarction in rats quantified with diffusion tensor MRI, American Journal of Physiology - Heart and Circulatory Physiology, 285 (2003), pp. H946-H954, https://doi.org/10.1152/ajpheart.00889.2002.

[7] H. Cheng And S. Torquato, Effective conductivity of periodic arrays of spheres with interfacial resistance, Proceedings: Mathematical, Physical and Engineering Sciences, 453 (1997), pp. 145-161, http://www.jstor.org/stable/52987.

[8] E. Fieremans, L. M. Burcaw, H.-H. Lee, G. Lemberskiy, J. Veraart, and D. S. Novikov, In vivo observation and biophysical interpretation of time-dependent diffusion in human white matter, NeuroImage, 129 (2016), pp. 414 - 427, https://doi.org/http://dx.doi. org/10.1016/j.neuroimage.2016.01.018, http://www.sciencedirect.com/science/article/pii/ S1053811916000240.

[9] D. Grebenkov, NMR survey of reflected brownian motion, Reviews of Modern Physics, 79 (2007), pp. 1077-1137, http://dx.doi.org/10.1103/RevModPhys.79.1077.

[10] D. Grebenkov, Laplacian eigenfunctions in NMR. I. A numerical tool, Concepts in Magnetic Resonance Part A, 32A (2008), pp. 277-301, http://dx.doi.org/10.1002/cmr.a.20117.

[11] D. S. Grebenkov, Laplacian eigenfunctions in NMR. II. Theoretical advances, Concepts Magn. Reson., 34A (2009), pp. 264-296, http://dx.doi.org/10.1002/cmr.a.20145.

[12] L. Greengard and J. Strain, A fast algorithm for the evaluation of heat potentials, Comm. Pure Appl. Math., 43 (1990), pp. 949-963.

[13] R. B. Guenther and J. W. Lee, Partial differential equations of mathematical physics and integral equations, Prentice Hall, Inglewood Cliffs, New Jersey, 1988.

[14] H. HADDAR, J.-R. LI, AND S. Schiavi, A Macroscopic Model for the Diffusion MRI Signal Accounting for Time-Dependent Diffusivity SIAM Journal on Applied Mathematics, 76 (2016), pp. 930-949, https: //doi.org/10.1137/15M1019398, http://dx.doi.org/10.1137/15M1019398, https: //arxiv.org/abs/http://dx.doi.org/10.1137/15M1019398.

[15] D. Hasselman and L. F. Johnson, Effective thermal conductivity of composites with interfacial thermal barrier resistance, Journal of Composite Materials, 21 (1987), pp. 508515.

[16] V. Kenkre, Simple solutions of the Torrey-Bloch equations in the NMR study of molecular diffusion, Journal of Magnetic Resonance, 128 (1997), pp. 62-69, http://dx.doi.org/10. 1006/jmre.1997.1216.

[17] V. G. KISELEV, The cumulant expansion: an overarching mathematical framework for understanding diffusion nmr, Diffusion MRI: Theory, Methods, and Applications: Theory, Methods, and Applications, (2010), p. 152, http://books.google.com/books?hl= en\&amp;lr=\&amp;id=dbZCMePD52AC\&amp;oi=fnd\&amp;pg=PA152\&amp;dq=The+ cumulant+expansion:+an+overarching+mathematical+framework+for+understanding+ diffusion+NMR\&amp;ots=YI6BKXfFDr\&amp;sig $=$ WoLmTLw7ZGATkUe-g3EGbtfz79I.

[18] L. L. Latour, P. P. Mitra, R. L. Kleinberg, and C. H. Sotak, Time-dependent diffusion coefficient of fluids in porous media as a probe of surface-to-volume ratio, Journal of Magnetic Resonance, Series A, 101 (1993), pp. 342-346.

[19] L. L. Latour, K. Svoboda, P. P. Mitra, and C. H. Sotak, Time-dependent diffusion of water in a biological model system., Proceedings of the National Academy of Sciences, 91 (1994), pp. 1229-1233, http://www.pnas.org/content/91/4/1229.abstract.

[20] M. LAzAR, Mapping brain anatomical connectivity using white matter tractography, NMR Biomed., 23 (2010), pp. 821-835, http://dx.doi.org/10.1002/nbm.1579.

[21] D. Le Bihan and H. Johansen-Berg, Diffusion MRI at 25: Exploring brain tissue structure 
and function, NeuroImage, 61 (2012), pp. 324-341, http://www.sciencedirect.com/science/ article/pii/S1053811911012857.

[22] D. LeBihan, S.-I. Urayama, T. Aso, T. Hanakawa, and H. Fukuyama, Direct and fast detection of neuronal activation in the human brain with diffusion mri, PNAS, 103 (2006), pp. 8263-8268, http://www.pnas.org/content/103/21/8263.abstract.

[23] J.-R. LI, D. CAlhoun, C. Poupon, AND D. L. Bihan, Numerical simulation of diffusion MRI signals using an adaptive time-stepping method, Physics in Medicine and Biology, 59 (2014), p. 441, http://stacks.iop.org/0031-9155/59/ $\mathrm{i}=2 / \mathrm{a}=441$.

[24] J.-R. Li And L. GReEngard, High order accurate methods for the evaluation of layer heat potentials, SIAM J. Sci. Comput., 31 (2009), pp. 3847-3860, https://doi.org/10.1137/ 080732389, http://dx.doi.org/10.1137/080732389.

[25] S. E. Maier, Y. Sun, And R. V. Mulkern, Diffusion imaging of brain tumors, NMR Biomed., 23 (2010), pp. 849-864, http://dx.doi.org/10.1002/nbm.1544.

[26] P. P. Mitra, P. N. Sen, and L. M. Schwartz, Short-time behavior of the diffusion coefficient as a geometrical probe of porous media, Phys. Rev. B, 47 (1993), pp. 8565-8574, https:// doi.org/10.1103/PhysRevB.47.8565, http://link.aps.org/doi/10.1103/PhysRevB.47.8565.

[27] P. P. Mitra, P. N. Sen, L. M. Schwartz, and P. Le Doussal, Diffusion propagator as a probe of the structure of porous media, Physical review letters, 68 (1992), pp. 3555-3558.

[28] M. E. Moseley, J. Kucharczyk, J. Mintorovitch, Y. Cohen, J. Kurhanewicz, N. DeruGin, H. AsGari, AND D. Norman, Diffusion-weighted MR imaging of acute stroke: correlation with T2- weighted and magnetic susceptibility-enhanced MR imaging in cats, AJNR Am J Neuroradiol, 11 (1990), pp. 423-429, http://www.ajnr.org/cgi/content/ abstract $/ 11 / 3 / 423$.

[29] C. H. Neuman, Spin echo of spins diffusing in a bounded medium, The Journal of Chemical Physics, 60 (1974), p. 4508, http://link.aip.org/link/?JCPSA6/60/4508/1.

[30] D. S. Novikov, E. Fieremans, J. H. Jensen, and J. A. Helpern, Random walks with barriers, Nat Phys, 7 (2011), pp. 508-514, http://dx.doi.org/10.1038/nphys1936.

[31] D. S. Novikov, J. H. Jensen, J. A. Helpern, And E. Fieremans, Revealing mesoscopic structural universality with diffusion, Proceedings of the National Academy of Sciences, (2014), https://doi.org/10.1073/pnas.1316944111, http://www.pnas.org/ content/early/2014/03/21/1316944111.abstract, https://arxiv.org/abs/http://www.pnas. org/content/early/2014/03/21/1316944111.full.pdf+html.

[32] D. S. Novikov And V. G. Kiselev, Effective medium theory of a diffusion-weighted signal, NMR in Biomedicine, 23 (2010), pp. 682-697, https://doi.org/10.1002/nbm.1584, http: //dx.doi.org/10.1002/nbm.1584.

[33] N. Pyatigorskaya, D. Le Bihan, O. Reynaud, and L. Ciobanu, Relationship between the diffusion time and the diffusion MRI signal observed at 17.2 tesla in the healthy rat brain cortex Magnetic Resonance in Medicine, (2013), pp. n/a-n/a, https://doi.org/10.1002/mrm. 24921, http://dx.doi.org/10.1002/mrm.24921.

[34] B. Robertson, Spin-echo decay of spins diffusing in a bounded region, Physical Review, 151 (1966), p. 273, http://prola.aps.org/abstract/PR/v151/i1/p273_1.

[35] D. Rohmer, A. Sitek, and G. T. Gullberg, Reconstruction and visualization of fiber and sheet structure with regularized tensor diffusion MRI in the human heart, Lawrence Berkeley National Laboratory Publication. LBNL-60277, (2006).

[36] E. O. Stejskal and J. E. Tanner, Spin diffusion measurements: Spin echoes in the presence of a time-dependent field gradient, The Journal of Chemical Physics, 42 (1965), pp. 288-292, http://dx.doi.org/10.1063/1.1695690.

[37] T. Sugahara, Y. Korogi, M. Kochi, I. Inushima, Y. Shigematu, T. Hirai, T. Okuda, L. Liang, Y. Ge, Y. Komohara, Y. Ushio, and M. TAKahashi, Usefulness of diffusion-weighted MRI with echo-planar technique in the evaluation of cellularity in gliomas, J. Magn. Reson. Imaging, 9 (1999), pp. 53-60, http://dx.doi.org/10.1002/(SICI) 1522-2586(199901)9:1〈53::AID-JMRI7)3.0.CO;2-2.

[38] A. Szafer, J. Zhong, And J. C. Gore, Theoretical model for water diffusion in tissues, Magn. Reson. Med., 33 (1995), pp. 697-712, http://dx.doi.org/10.1002/mrm.1910330516.

[39] S. Torquato and M. D. Rintoul, Effect of the interface on the properties of composite media, Phys. Rev. Lett., 75 (1995), pp. 4067-, http://link.aps.org/doi/10.1103/PhysRevLett.75. 4067.

[40] Y. Tsushima, A. Takahashi-Taketomi, and K. Endo, Magnetic resonance (MR) differential diagnosis of breast tumors using apparent diffusion coefficient (ADC) on 1.5-t, J. Magn. Reson. Imaging, 30 (2009), pp. 249-255, http://dx.doi.org/10.1002/jmri.21854.

[41] D. S. Tuch, T. G. Reese, M. R. Wiegell, and V. J. Wedeen, Diffusion MRI of complex 
neural architecture, Neuron, 40 (2003), pp. 885-895.

[42] S. Warach, D. Chien, W. Li, M. Ronthal, and R. R. Edelman, Fast magnetic resonance diffusion-weighted imaging of acute human stroke, Neurology, 42 (1992), pp. 1717-, http: //www.neurology.org/cgi/content/abstract/42/9/1717. 